\title{
Proximity Effect in Crystalline Framework Materials: Stacking-Induced Functionality in MOFs and COFs
}

\author{
Agnieszka Kuc,* Maximilian A. Springer, Kamal Batra, Rosalba Juarez-Mosqueda, \\ Christof Wöll, and Thomas Heine**
}

Metal-organic frameworks (MOFs) and covalent organic frameworks (COFs) consist of molecular building blocks being stitched together by strong bonds. They are well known for their porosity, large surface area, and related properties. The electronic properties of most MOFs and COFs are the superposition of those of their constituting building blocks. If crystalline, however, solid-state phenomena can be observed, such as electrical conductivity, substantial dispersion of electronic bands, broadened absorption bands, formation of excimer states, mobile charge carriers, and indirect band gaps. These effects emerge often by the proximity effect caused by van der Waals interactions between stacked aromatic building blocks. Herein, it is shown how functionality is imposed by this proximity effect, that is, by stacking aromatic molecules in such a way that extraordinary properties emerge in MOFs and COFs. After discussing the proximity effect in graphene-related materials, its importance for layered COFs and MOFs is shown. For MOFs with well-defined structure, the stacks of aromatic building blocks can be controlled via varying MOF topology, lattice constant, and by attaching steric control units. Finally, an overview of theoretical methods to predict and analyze these effects is given, before the layer-by-layer growth technique for well-ordered surface-mounted MOFs is summarized.

\section{Introduction}

Molecular framework materials, including metal-organic frameworks (MOFs), ${ }^{[1-3]}$ coordination polymers, ${ }^{[4]}$ and covalent organic frameworks (COFs), ${ }^{[5,6]}$ provide an intriguing bridge between chemistry and solid-state physics. They are composed of molecular units that may carry the whole range of functional groups known to chemistry. These molecular building blocks are stitched together by strong bonds. This setup provides much higher chemical, thermal, and mechanical stability as compared to molecular crystals and, thus, allows the formation of large pores which can reach up to $10 \mathrm{~nm}$ in diameter, ${ }^{[7-10]}$ as well as extremely large internal surface areas (see, e.g., ref. [9] and references therein). These unique properties have motivated intense MOF and COF research during the past years: They allow high gas uptake capacities and, thus, application in methane and hydrogen storage. ${ }^{[11,12]}$

Dr. A. Kuc, M. A. Springer, Prof. T. Heine

Helmholtz-Zentrum Dresden-Rossendorf

Institut für Ressourcenökologie

Forschungsstelle Leipzig, Permoserstr. 15, 04318 Leipzig, Germany

E-mail: a.kuc@hzdr.de

M. A. Springer, K. Batra, Prof. T. Heine

Theoretical Chemistry

Technische Universität Dresden

Bergstr. 66c, 01062 Dresden, Germany

E-mail: thomas.heine@tu-dresden.de

Dr. R. Juarez-Mosqueda

Department of Chemical Engineering

University of Pittsburgh

Pittsburgh, PA 15261, USA

Prof. C. Wöll

Karlsruhe Institute of Technology (KIT)

Institute of Functional Interfaces (IFG)

Hermann-von-Helmholtz-Platz 1, 76344 Eggenstein-Leopoldshafen, Germany

The ORCID identification number(s) for the author(s) of this article can be found under https://doi.org/10.1002/adfm.201908004.

(C) 2020 The Authors. Published by WILEY-VCH Verlag GmbH \& Co. KGaA, Weinheim. This is an open access article under the terms of the Creative Commons Attribution License, which permits use, distribution and reproduction in any medium, provided the original work is properly cited.

DOI: $10.1002 / \mathrm{adfm} .201908004$
Coupling these properties, intrinsic to porous materials, with molecular functionalities integrated into nodes and linkers can yield to multifunctionality: selective uptake, $\mathrm{CO}_{2}$ capture, ${ }^{[13,14]}$ hydrogen isotope separation, ${ }^{[15,16]}$ as well as switching permeance and selectivity via optical ${ }^{[17]}$ or electrical ${ }^{[18]}$ switching. Catalysis also profits from the presence of large pores and channels, which allow transport of reactants and products, and, if coupled with functional groups, turns the voids in these materials into selective nanoreactors. ${ }^{[19]}$ Even though the International Union of Pure and Applied Chemists (IUPAC) recommendations for nomenclature explicitly specifies that these materials are not necessarily crystalline, ${ }^{[20]}$ high crystal order is observed for many of them.

Crystallinity allows high-quality structural analysis by experimental methods, for example, via X-ray diffraction (XRD). As a consequence, theoretical work can be carried out in a straightforward fashion and can then be compared directly to experimental results, thus, allowing for a direct validation of computational approaches. The well-defined structure also makes a proper physicochemical characterization of these materials possible and provides, thus, the basis for the high level of understanding of their structure and electronic structure. At the same time, crystal order is the reason for solid-state effects that are caused by the translational symmetry, such as magnetic order, indirect band gaps, and ballistic charge transport. ${ }^{[21]}$ As in other 
crystalline solids, defects play a critical role for certain properties, including catalytic activity, electronic and optical properties; thus, a proper characterization of defect types and their density is crucial. ${ }^{[2,23]}$ This point is particularly important, as the properties discussed below are a direct consequence of the crystallinity of the framework materials.

Intrinsic magnetic order suffers from the large distances between the spin centers in a MOF and the resulting weak couplings in the order of a few meVs. Thus, they are only observed at cryogenic temperatures. ${ }^{[24-26]}$ Ballistic transport is typically hindered either by the large effective masses of the typically dispersionless bands or by the chemical composition of the frameworks, where non-carbon centers (e.g., oxygen, boron, or nitrogen) can effectively block electron conjugation. Only recently, ballistic transport, facilitated by electron conjugation, has been demonstrated in layers of two-dimensional (2D) MOFs and COFs. ${ }^{[27-30]}$ There is, however, a more subtle way to implement strong band dispersion in crystalline molecular framework materials: Controlled stacking of aromatic molecules, incorporated into the materials as linkers or as pillars with suitable intermolecular distance, is subject to the proximity effect, more precisely, the molecules interact via $\pi$-stacking, which causes strong alterations of the electronic structure of the framework materials. The proximity effect is the basis of the recent breakthrough in van der Waals physics of 2D materials (see, e.g., refs. [31-40]). While this type of stacking is rather obvious for the class of layered $\mathrm{COFs}^{[41]}$ with atomically thin layers and with aromatic connectors or linkers, it can also be achieved in MOFs with suitable crystal structure. ${ }^{[42,43]}$

A further option of MOFs and, to some extent, also COFs is to grow superstructures by applying layer-by-layer (lbl) procedures (MOF-on-MOF) and to fabricate structurally well-defined organic/organic heterointerfaces. ${ }^{[4,45]}$

In this Progress Report, we elaborate the impact of the proximity effect in MOFs and COFs with suitable crystal structures. We will first discuss the importance of the proximity effect for the case of graphene, which serves as role model for the stacked aromatic moieties in MOFs and COFs. In order to reach a wide audience with background in chemistry, we will introduce key concepts of solid-state physics, when appropriate. We will then approach the closest relatives to graphene, stacked layered COFs, before we explore pathways to tailor these interactions by means of crystal structure and functional side groups in both layered and three-dimensional (3D) MOFs. The conclusions include our perspective of further exploiting the proximity effect in crystalline porous framework materials and, thus, exploiting the fusion of most recent advances in the condensed matter physics and materials chemistry. In the Section 7, we give an overview of theoretical approaches, rationally design and to computationally explore these materials, and summarize the lbl growth technique to synthesize surface-mounted MOFs with controlled stacks of aromatic linker molecules.

\section{Stacking of Graphene Layers}

In order to understand the potential impact of stacking of aromatic moieties in molecular framework materials, it is useful to

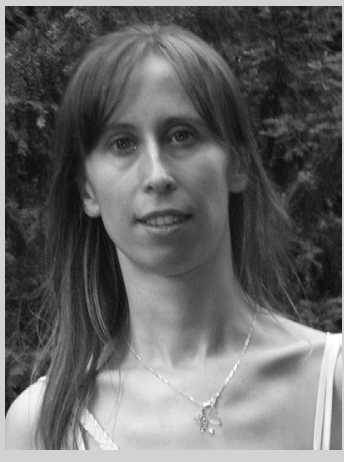

Agnieszka Kuc graduated from Wroclaw University in 2003 with an M.Sc. in physical chemistry. She completed her Ph.D. in chemistry under the supervision of Prof. Gotthard Seifert at TU Dresden in 2008. She habilitated in Physics in 2018 under mentoring of Prof. Thomas Heine at Jacobs University Bremen. She is currently working as a group leader at Helmholtz-Zentrum DresdenRossendorf. Her research interests include, among others, electronic structure simulations and vibrational properties of 2D inorganic and hybrid materials, such as transitionmetal dichalcogenides, conjugated polymers, metal- and covalent organic frameworks, and perovskites.

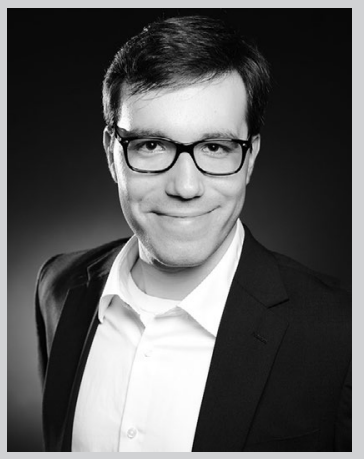

Maximilian A. Springer received his B.Sc. (2015) and M.Sc. (2018) in chemistry from Leipzig University, Germany. During his studies, he accomplished research stays at Memorial University of Newfoundland, St. John's, Canada (2015) and Monash University, Melbourne, Australia (2016). He pursues his Ph.D. studies under supervision of Thomas Heine and Agnieszka Kuc at the Leipzig research site of Helmholtz-Zentrum DresdenRossendorf, Institute of Resource Ecology and at TU Dresden. His current research focuses on the relation of electronic structure and topology in 2D polymers.

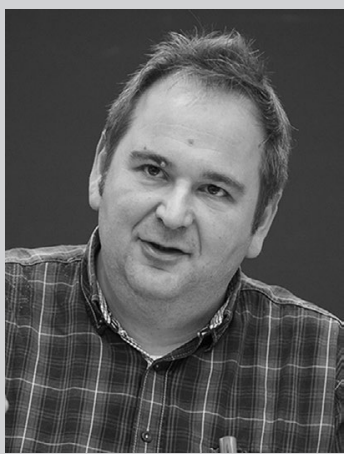

Thomas Heine graduated in physics from TU Dresden under the guidance of Gotthard Seifert. After various research stages he obtained the venia legend in physical chemistry at TU Dresden. In 2008, he was appointed as Associate Professor of Theoretical Physics at Jacobs University, and moved as Professor of Theoretical Chemistry to Leipzig University in 2015. Since 2018, he holds the Chair of Theoretical Chemistry at TU Dresden and leads the Theoretical Chemistry group at the Leipzig branch of Helmholtz-Zentrum Dresden-Rossendorf. His research interests include framework materials, 2D crystals, theoretical spectroscopy, and method development for theoretical materials science. 
discuss the proximity effect in graphene. Graphene ${ }^{[46]}$ is by far the most widely studied 2D material to date and, at the same time, it can be considered as the prototype of a $2 \mathrm{D}$ polymer.

The extraordinary properties of graphene are distinct from those of its parental 3D system, graphite: massless Dirac fermions, exceptionally high electrical-conductivity, long-distance spin-transport, and half-integer quantum Hall effect. ${ }^{[47,48]}$ The Dirac points (Figure 1a) in the Brillouin zone of graphene, situated at each $K$ point, cause these very interesting features.

Stacking two or more single graphene layers on top of one another may, however, change most of the electronic properties of graphene. In the solid state, Bernal graphite is the most common form, ${ }^{[49]}$ where the graphene layers are stacked in the $A B A B$ fashion, such that centers of sixfold rings of one layer are situated on top of carbon atoms in the second layer, forming the so-called staggered structure. Bernal graphite requires four carbon atoms in the unit cell, which results in four bands at the $K$ point of the Brillouin zone close to the Fermi level, however, they are now parabolic rather than linear, and, thus, give rise to massive electrons in a zero-gap semiconductor (Figure 1b; see also refs. [50,51]). On the other hand, an almost linear (more accurately, a parabolic dispersion with very large curvature) and very small effective mass can be seen at the $H$ point of the Brillouin zone, with the Fermi level slightly below the top of the valence band and a band gap of about $5 \mathrm{meV},{ }^{[52]}$ thus, a few orders of magnitudes larger than in graphene.

In a hypothetical structure of eclipsed graphite (AAAA stacking of layers, Figure 1c), an intriguing electronic structure emerges. Two atoms per unit cell and one layer are necessary to represent the smallest possible 3D structure. There are two nearly linear bands at the $K$ point close to the Fermi level, similar to graphene. However, the two Dirac points without band gaps at $K$ and $H$ points are above and below the Fermi level, respectively. Thus, the $\pi$ and $\pi^{*}$ bands are not symmetric with respect to the Fermi level. This demonstrates that the proximity effect depends both on the distance between the layers and on the mutual shift of the crystal planes.

Even more strikingly, strong effects are observed if two adjacent graphene layers are twisted with respect to each other. The beautiful moiré structures of the lattice are reflected also in the electronic structure (in presence of magnetic field resulting in the Hofstadter butterfly). ${ }^{[39]}$ Most notably, in 2018, Cao et al. ${ }^{[31,56]}$ showed that at a magic twist angle of $1.05^{\circ}$, correlation is strongly enhanced and a Mott insulating state is formed,

\section{Graphite}
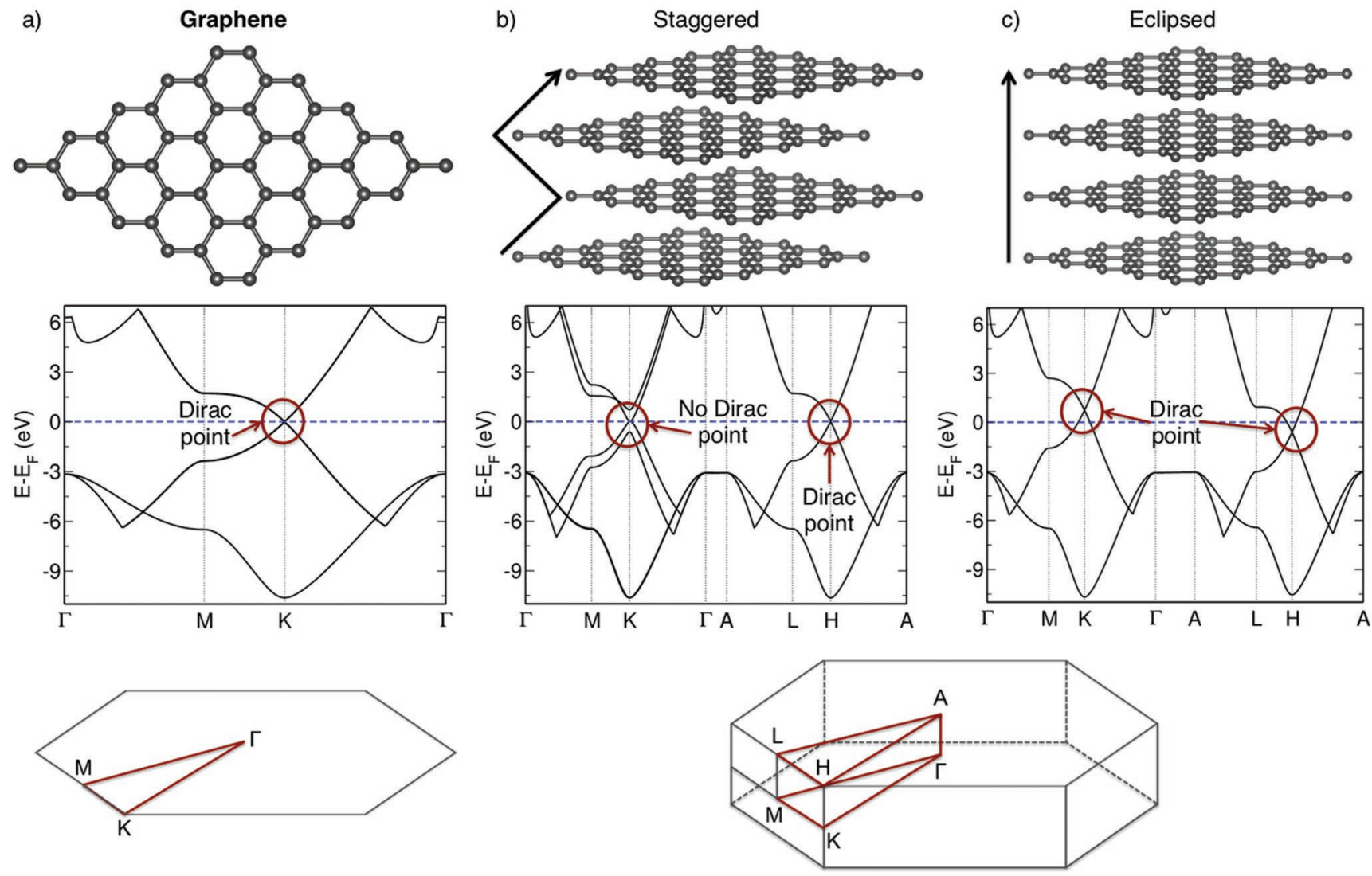

Figure 1. (Top) Structures, (middle) electronic structures, and (bottom) Brillouin zones of a) graphene, b) staggered graphite ( $A B A B$ stacking, Bernal structure $\left.{ }^{[49]}\right)$, and c) eclipsed graphite (AAAA stacking, hypothetical). Single layer of graphene, which exhibits Dirac points (linear dispersion relation) at $K$ points and a band gap of about $1 \mu \mathrm{eV},{ }^{[53]}$ changes its electronic properties when stacked differently into 3D structures: $A B A B$ graphite has quadratic and nearly linear dispersion relations at the $K$ and $H$ points, respectively, and band gap at $H$ of 5 meV ${ }^{[52]}$; AAAA graphite has two Dirac points (at $K$ and $H$ points) above and below Fermi level (blue dashed horizontal lines). Simulations at the DFT/PBE-D3(BJ) level of theory with TZP basis set and spin-orbit coupling as implemented in AMS/BAND software. ${ }^{[54]}$ Pictures of structures made with VESTA. ${ }^{[55]}$ 

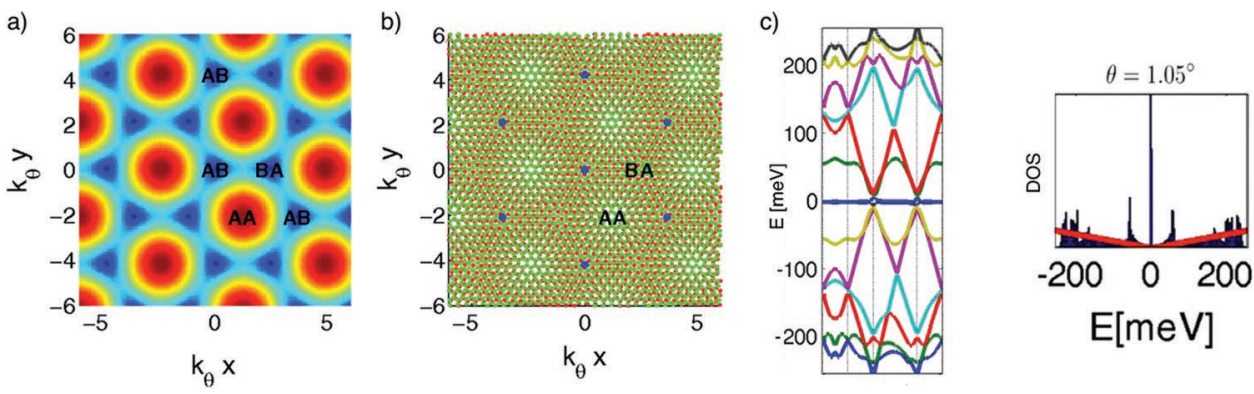

Figure 2. $a, b)$ Moiré period and pattern obtained from two graphene layers twisted with respect to one another. High symmetry local stackings are denoted ( $A B$ stacking in (b) denoted with blue dots). a) Smallest positive energy of the interlayer Hamiltonian. The energy vanishes for local $A B$ or $B A$ coordination and reaches a maximum for local $A A$ coordination. c) Energy dispersion for the 14 bands closest to the Dirac point plotted along the k-space trajectory $K-\Gamma-K_{1}^{\prime}-K_{2}^{\prime}{ }_{2} K$ and the corresponding density of states (DOS). Adapted with permission. ${ }^{[57]}$ Copyright 2011 , National Academy of Sciences.

which results in a superconducting state. This effect was shown initially in 2011 by Bistritzer and MacDonald, ${ }^{[57]}$ who reported that the Dirac-point velocity vanishes at the twist angle of $1.05^{\circ}$ and that this effect is accompanied by a very flat moiré band, which results in a sharp peak in the Dirac-point density of states (Figure 2). The difference between flat, conjugated bands, and dispersionless bands should be noted here: the former results from the symmetry of the lattice (e.g., kagome) with extended $\pi$ conjugation, while the latter indicates localized, non-interacting states without electron conjugation.

This short summary of electronic properties of graphene, bilayer graphene, and graphite illustrates the importance of the proximity effect: Even though the graphene layers are subject to only weak van der Waals forces, which leave the atomistic structure essentially unaffected, the interlayer interaction has significant impact on the electronic structure, which is so strong that it can even cause the electronic phase transition from a zerogap semiconductor to a superconductor. ${ }^{[31]}$ Similar stackings are present in layered COFs and in MOFs with suitable geometries. The impact of the proximity effect in these materials will be discussed in the next chapters.

\section{Stacking of 2D Covalent Organic Frameworks}

In 2005, Côte and coworkers ${ }^{[5]}$ introduced a new family of layered and porous materials which are referred to as covalent organic frameworks (COFs). In these systems, organic linker molecules are connected via covalent bonds with connectors, formed, for example, by oxygen and boron atoms (see Figure 3b for building blocks of COF-5). Regular frameworks, formed by stitching together these linkers and connectors, can have honeycomb topology within each layer, as in the case of COF-5 (Figure 3d), but are not restricted to this topology. For example, using porphyrins (PP) as tetratopic organic linkers leads to a square topology. ${ }^{[5,59]}$ Note that even though these materials are referred to as "2D COFs," in the literature, they are in fact rather layered materials, where single 2D sheets form 3D structures with porous channels normal to the basal planes. Nowadays, many different COF systems have been synthesized and investigated, and several reviews or perspective articles are available in the literature. ${ }^{[60-62]}$

Here, we would like to focus on the aspect of layer stacking and its effect on the electronic properties of selected COFs. In
2011, we have calculated the stacking order of a few layered COFs from first principles. ${ }^{[63,64]}$ We focused especially on the structures of COF-1, $-5,-6$, and -8 . Back in 2011, the experimentally reported layer stacking of these systems was either $A A A A$ (eclipsed) (cf. Figure $3 e$, top) or $A B A B$ (staggered). We showed that these systems are considerably more energetically stable (Figure 3a) if their stacking arrangement is either serrated $\left(A A^{\prime} A A^{\prime}\right.$, that is, the sixfold rings in the linkers and connectors are stacked as in Bernal graphene, cf. Figure $3 \mathrm{~g}$ top for COF-5) or inclined ( $A A^{\prime} A^{\prime \prime} A^{\prime \prime \prime}$, cf. Figure $3 f$, top). In these arrangements, the layers are shifted with respect to one another by about one $\mathrm{C}-\mathrm{C}$ bond length, $\approx 1.4 \AA$, compared with the eclipsed stacking. Note that many other stackings, possibly random ones, are possible. Our calculated powder X-ray diffraction (PXRD) patterns fit very well to the experimental ones (Figure 3c). We also showed that the proposed stackings of layers affect the pore geometries. Pores in COFs are the functional regions and we calculated that the inclined and serrated arrangements account for an increase in the surface area by $6 \%$, estimated for the interaction with $\mathrm{He}$, or $3 \%$ for the interaction with $\mathrm{N}_{2}$, compared with eclipsed stacking. Moreover, the polarity of pores increases for the two stackings, because both oxygen and boron atoms are exposed to the pore surface. After our report on the stacking structure of COFs, the proposed stacking arrangements, especially the serrated one, were commonly adapted in the community. ${ }^{[65]}$ In 2017, Fan et al. ${ }^{[66]}$ demonstrated for the first time inclined stacking of layers in 2D COFs by introduction of steric substituents between the layers.

We have simulated the electronic band structure of single layer of COF-5, as an exemplary 2D COF structure, and compared it to these of bulk COF-5 with different stackings: eclipsed, inclined, and serrated (see Figure 3 bottom panels for full band structure and zoom-ins of conduction and valence bands). The band structure of the single layer of COF-5 exhibits almost flat bands when compared to the 3D stackings. However, close examination of the valence and conduction bands in the vicinity of the Fermi level of the COF-5 single layer reveals a typical signature of the so-called (Archimedean) kagome lattice (Figure 3d, conduction band minimum (CBM) zoom). In the valence band (Figure $3 \mathrm{~d}$, valence band maximum (VBM) zoom), these are two bands of linear dispersion, crossing at the $K$ point, similar to graphene. These bands are also sandwiched between two flat bands, forming so-called ruby bands. Thus, the top of valence band is very flat throughout the Brillouin zone. 


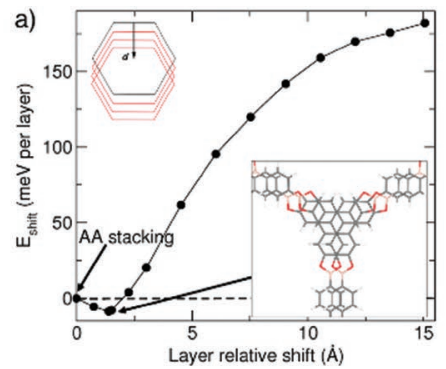

b) Building Blocks

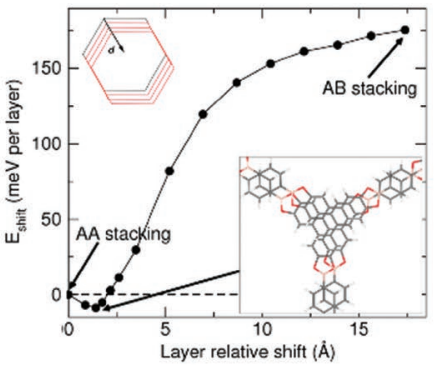<smiles>Oc1cc2c3cc(O)c(O)cc3c3cc(O)c(O)cc3c2cc1O</smiles><smiles>OB(O)c1ccc(B(O)O)cc1</smiles>

d) Monolayer
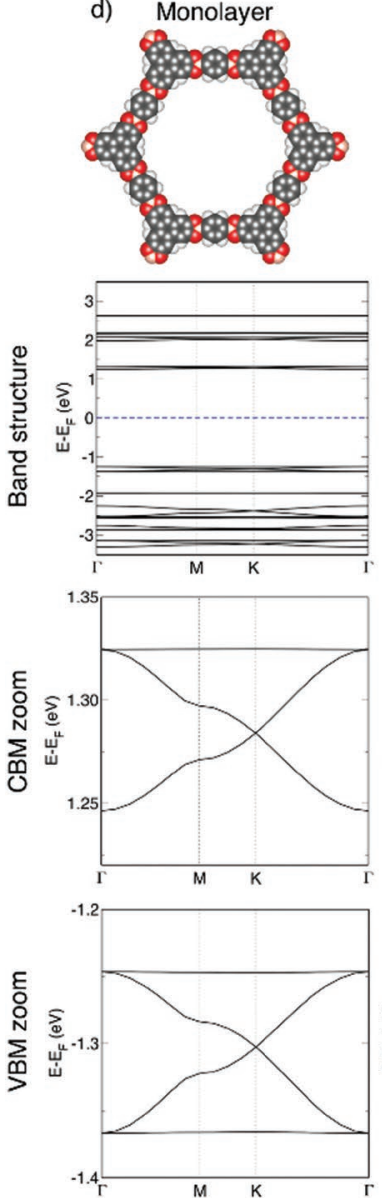

e) Eclipsed
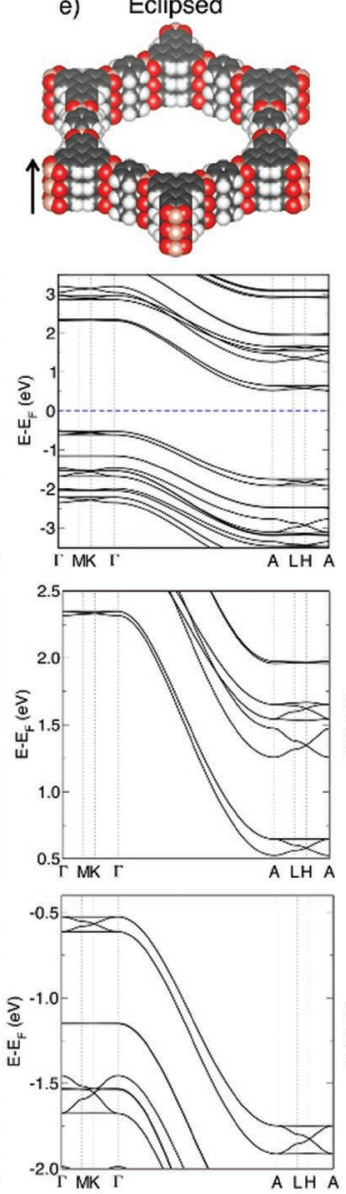

c)
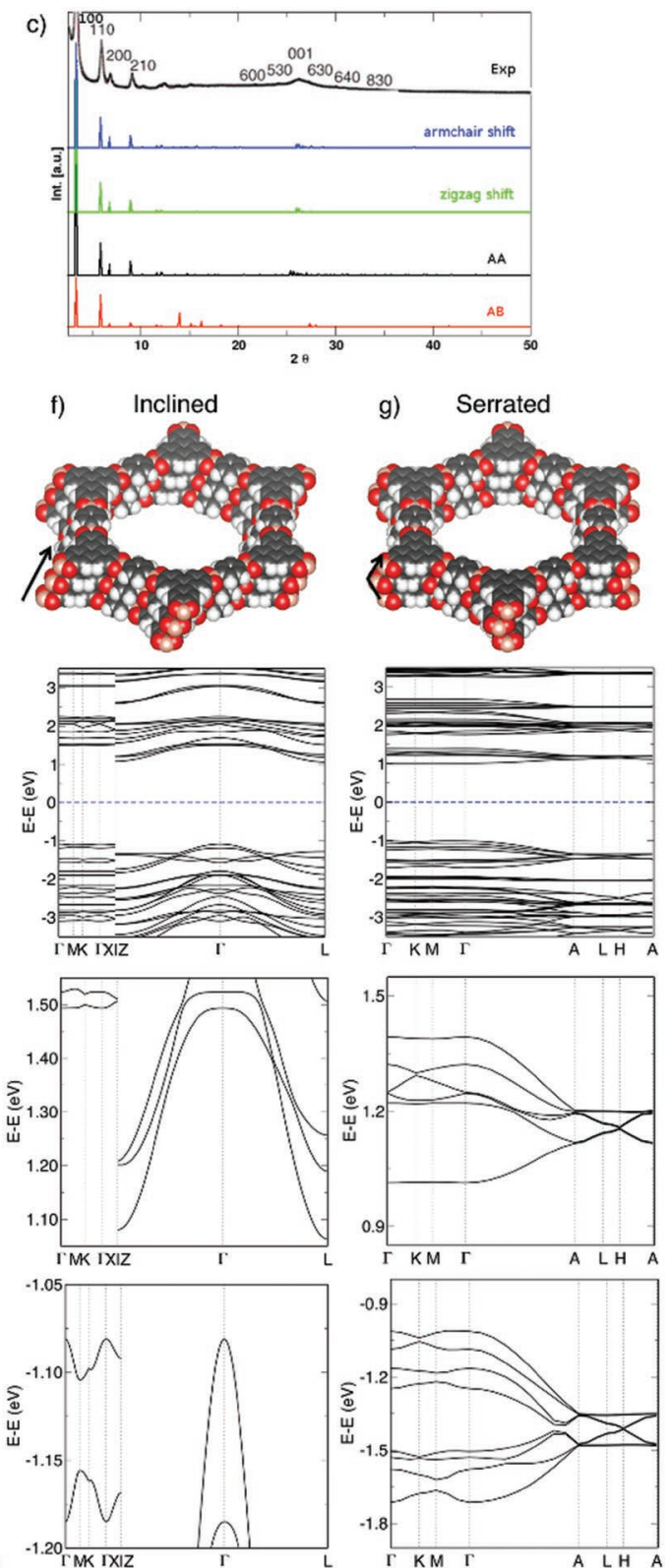

Figure 3. a) Relative energy when shifting layers of COF-5 with respect to one another in armchair (left) and zigzag (right) directions. The energies of the two high symmetry stackings $(A A A A$ and $A B A B)$ are indicated. The low-energy minimum corresponds to the equilibrium structures, shown in the insets. b) Building blocks of COF-5. c) Experimental and calculated PXRD patterns of all the stacking arrangements. $d-g$ ) (Top) structures and (bottom) electronic band structures (lowest two panels show zoom-ins of conduction band maximum [CBM] and valence band minimum [VBM]) of a) monolayer $(1 \mathrm{~L}), \mathrm{b})$ eclipsed $(A A A A), \mathrm{c})$ inclined $\left(A A^{\prime} A^{\prime \prime} A^{\prime \prime}\right)$, and d) serrated $\left(A A^{\prime} A A^{\prime}\right)$ stackings of COF-5. Single layer COF-5 has fairly flat bands close to the Fermi level (blue dashed horizontal lines). Prominent dispersion of bands in the direction perpendicular to the layers can be observed upon stacking single layer to multilayer COF-5, the strongest in case of AAAA (eclipsed) stacking. Calculations performed on the optimized structures from ref. [63] at the DFT/PBE level of theory with TZP basis set as implemented in AMS/BAND software. ${ }^{[54]}$ a,c) Adapted with permission. ${ }^{[63]}$ Copyright 2011 , Wiley. Pictures of structures made with VESTA. ${ }^{[5]}$ 
The conduction bands are a bit more disperse, with minimum at the $\Gamma$ point. The single layer of COF-5 is, thus, a direct-gap semiconductor with a band gap of about $2.7 \mathrm{eV}$. Our results are in good agreement with previously reported theoretical work of Liu et al., ${ }^{[67]}$ who obtained a band gap of $2.5 \mathrm{eV}$ at a similar level of theory.

Stacking of COF-5 layers into a 3D structure with different arrangements (Figure $3 \mathrm{~d}-\mathrm{f}$ ) changes electronic properties of the materials. While the eclipsed stacking keeps almost unchanged in-plane signatures of the kagome lattice, interlayer interactions induce strong band dispersion in the direction perpendicular to the layers. The band dispersion between $\Gamma$ and $A$ points in the Brillouin zone reaches almost $1 \mathrm{eV}$. As discussed above, this stacking is, however, energetically unfavorable. The two most stable high-symmetry structures of COF-5, inclined and serrated, strongly alter the electronic structure of the monolayer. In both cases, the in-plane kagome characteristic is no longer present along the $\Gamma-K-M-\Gamma$ path. For serrated case, some kagome signature is visible for $A-L-H-A$ path, however, these bands are not forming the band edges, as in the monolayer or eclipsed form. Out-of-plane dispersion is also present, but smaller than in the eclipsed stacking. For the serrated case, it is less than $0.5 \mathrm{eV}$. Band gap values and characters also change compared with the monolayer case: For the eclipsed structure, we calculated an indirect band gap of about $1.3 \mathrm{eV}$ between $\Gamma$ and $A$ points, for the inclined structure, we obtained an indirect band gap of about $2.2 \mathrm{eV}$ between $X$ and $\Gamma$ points, while serrated stacking gives also an indirect band gap of about $2.2 \mathrm{eV}$ between $M$ and $\Gamma$ points. Band dispersion is of course desirable for nanoelectronic applications, because curved bands at the band edges result in light charge carriers (holes and electrons). While COF-5 single layer offers light electrons and heavy holes, which is interesting for production of short transport channel devices, the stacking introduces also light holes, which could be interesting for standard transistor applications. This survey on COF-5 shows that, just as in graphene, the proximity effect has a strong influence on the electronic structure of molecular framework materials. After the reports of COF-1 and COF-5, many routes to form $2 \mathrm{D}$ COFs have been developed. While a comprehensive summary of the literature would not fit into the scope of this article, we highlight here the most important steps that are crucial for the development of van der Waals science in 2D COFs. First, a large variety of coupling reactions has been developed in the field of 2D polymers (the term 2D polymers is commonly used for single layers of 2D COFs). We show a selection in Figure 4.

Second, the large diversity of aromatic structures offers, besides honeycomb ${ }^{[5]}$ and square, ${ }^{[58,59]}$ also many other structural forms, including hexagonal ${ }^{[68,69]}$ and kagome. ${ }^{[70-73]}$ For detailed reviews, see refs. [74-77]. Moreover, even the symmetry of the pores can be controlled by proper choice of linkers. ${ }^{[78]}$ This illustrates that there is no doubt that the potential structures offered by 2D COFs significantly exceed those of dense layered materials that are available in nature or have been synthesized. [79-81]

Third, in order to enable ballistic electron transport also inside the individual COF layers, it is important to remove the a)<smiles>Cc1ccc(I)cc1</smiles>

$\mathrm{Cu}$
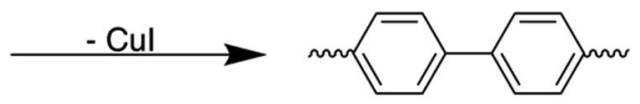

b)

c)<smiles>O=Cc1c(O)c(C=O)c(O)c(C=O)c1O</smiles>

d)<smiles>[R]N</smiles>

$+$

$\mathrm{BX}_{3}$

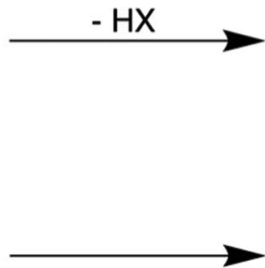<smiles>[R]B1OB([R])OB([R])O1</smiles><smiles>NC=C1C(=O)C(=CN)C(=CN)C1=O</smiles><smiles>[Y]B1NB([Y])N([R])B([Y])N1[R]</smiles><smiles>[R]c1nc([R])nc([R])n1</smiles>

Figure 4. Simplified schemes illustrating important coupling reactions for the formation of 2D polymers and 2D COFs. a) Ullmann coupling, b) boron esterification, c) Knoevenagel-like condensation, d) borazine formation, and e) nitrile cyclotrimerization. 
electron scattering centers, which typically are the atoms at the coupling sites. If they are perturbing the conjugation in the COF, electrons cannot flow and the electronic system is not much different to that of a molecular crystal. As shown in the groups of Jiang and Feng, fully conjugated 2D COFs are possible, for example, if aromatic building blocks are coupled by using the Knoevenagel reaction. ${ }^{[27,30]}$ These works demonstrate that 2D COFs with fully conjugated electronic $\pi$-system can be realized in experiments, which means that it is possible to construct high-mobility single layer 2D COF semiconductors. It remains to be explored which consequences the proximity effect has, if these 2D COFs are stacked upon one another, both in homogeneous and heterogeneous stacks.

To date, a huge variety of 2D COF structures has been synthesized and studied, for example, in the direction of photoactivity and photosensitivity. ${ }^{[61,78,82-87]}$ However, electronic structures are seldom discussed and, even then, mostly only single layers are considered. ${ }^{[60,88-90]}$ Thomas et al. ${ }^{[60,90]}$ investigated electronic structures of $2 \mathrm{D} \pi$-conjugated polymers with three- and four-arm connections, which correspond to kagome and Lieb (Archimedean) lattices, and explained occurrence of flat and disperse bands. However, no stacking was taken into account. On the other hand, Er et al. ${ }^{[88]}$ reported an excellent carrier mobility and photoconductivity along the vertical direction of a DA-COF (D, donor; A, acceptor). The authors found that these properties depend on the number of layers and the stacking of layers. The conduction was achieved by electron hopping between layers, in which the donor and the acceptor groups were aligned with respect to each other. This arrangement would correspond to the AAAA stacking discussed above. In other works on COF systems available in the literature, some of the COFs were reported to have high charge carrier mobilities, as in the case of COF-366 and COF-66, with covalently linked PP units offering extended planar $\pi$-electron system. ${ }^{[91]}$

\section{Layered, Atomically Thin Metal-Organic Frameworks}

MOFs $^{[1,2,92,93]}$ are prototypes of the reticular chemistry concept, in which well-defined building blocks are stitched together to form porous 3D frameworks. In the simplest case, two building blocks are used, linkers (typically di- or higher-topic organic molecules) and connectors (typically metal ions or metal/oxo clusters). To date, a huge variety of MOFs has been synthesized and characterized, with the most frequent applications in the fields of gas adsorption and separation, sensing, drug-release, proton conductance in fuel cells, catalysis, water splitting, and many others. ${ }^{[94,95]}$ In contrast to COFs, where the 2D versions are more prominent than their 3D counterparts, 2D MOFs with atomically thin layers coupled by van der Waals interactions are-to date-rather exotic and have been reported in a few cases only. ${ }^{[24,96-102]}$ However, the instances of such layered system reported so far promise an interesting bridge between chemistry and physics, offering materials with interesting electronic and magnetic properties.

Similar to COFs, and even sometimes better, MOFs also offer extended 2D $\pi$-conjugation. ${ }^{[103]}$ For example, a recent paper by Yang et al. ${ }^{[2]}$ reports on a semiconducting MOF magnet. $\mathrm{K}_{3} \mathrm{Fe}_{2}\left[\mathrm{PcFe}-\mathrm{O}_{8}\right]$ (Pc, phthalocyanine) exhibits spontaneous magnetization and full in-plane $\pi$-d conjugation, which results in room temperature carrier mobility of about $15 \mathrm{~cm}^{2} \mathrm{~V}^{-1} \mathrm{~s}^{-1}$. The long-range magnetism arises from the magnetic coupling between iron centers via the $\pi$-electron system. This MOF exhibits strong out-of-plane band dispersion, however, different to many COFs and MOFs, it also shows inplane dispersion. The $\mathrm{K}_{3} \mathrm{Fe}_{2}\left[\mathrm{PcFe}-\mathrm{O}_{8}\right]$ MOF consists of layers being only one atom thick (Figure 5a). Such atomically thin planar MOFs, with extended in-plane $\pi$-conjugation, are currently found to be the most conductive frameworks known to date. ${ }^{\left[{ }^{97-99]}\right.}$ For example, in the case of $\mathrm{Ni}_{3} \mathrm{HITP}_{2}$ (HITP: 2,3,6,7,10,11-hexaiminotriphenylene; see Figure 5b), the highly $\pi$-stacked and extended conjugation of the network results in high electrical conductivity. ${ }^{[100]}$ This semiconducting MOF was initially investigated by Dinca and coworkers ${ }^{[97]}$ and later by Zeng and coworkers. ${ }^{[101]}$ It has an interlayer distance of about $3.3 \AA$ and a conductivity of $40 \mathrm{~S} \mathrm{~cm}^{-1}$ at room temperature.

In another MOF-like coordination polymer based on benzenehexadelenolate, $\left(\left[\mathrm{Cu}_{3}\left(\mathrm{C}_{6} \mathrm{Se}_{6}\right)\right]_{\mathrm{n}}\right)$, with the $\pi$-d conjugation extended in-plane, ${ }^{[102]}$ the layers are again stacked in the $A A^{\prime} A A^{\prime}$ fashion and have $\mathrm{Cu}$ metal centers in a square planar configuration (Figure 5c). The interlayer distance is about $3.6 \AA$, very close to that in graphite. The material exhibits metallic character, similar to many other atomically thin MOFs, and very high room-temperature conductivity of $110 \mathrm{~S} \mathrm{~cm}^{-1}$.

It should also be noted that, using sophisticated forms of MOF post-synthesis modification, MOFs can be transformed into COFs by crosslinking the primary linkers with suitable secondary linkers and then removing the metal ions form the lattice. ${ }^{[104,105]}$ This approach can also be used to fabricate effectively 2D COF structures. ${ }^{[106]}$

\section{Proximity Effect in Metal-Organic Frameworks: The SURMOF Showcase}

Several groups have introduced $\mathrm{lbl}$ methods to deposit MOF thin films on substrate, first, in 2007, Wöll and Fischer reported on the lbl route to MOF synthesis, ${ }^{[95,107,108]}$ and later Kitagawa and coworkers. ${ }^{[109]}$ Such surface-mounted MOFs are referred to as SURMOFs. They exhibit high crystallinity and can be investigated using virtually all surface science techniques, see also Section on SURMOFs synthesis in Section 7. SURMOF-2, being an isoreticular series based on MOF-2, ${ }^{[110,111]}$ is one of the simplest MOF architectures suited for $1 \mathrm{bl}$ growth. ${ }^{[7]}$ They are derived from MOF-2, a bulk framework material based on paddle-wheel units with four dicarboxylate groups and typically $\mathrm{Cu}^{2+}$ or $\mathrm{Zn}^{2+}$-dimers connected to ditopic organic linkers of different length, the shortest one being 1,4-benzene dicarboxylate. The length of the linkers determines the lattice constant and, thus, the pore size of the resulting SURMOFs, where up to $4 \mathrm{~nm}$ in diagonal have been reached up thus far. ${ }^{[7]}$ Layers of such SURMOFs form square lattices and, theoretically, could be stacked together in three different arrangements. The most symmetric P4 variant is the eclipsed stacking with linkers and connectors in one layer directly on top of linkers and connectors in another layer (Figure 6a). This stacking is found in all SURMOF-2 derivatives discussed in this chapter and leads to 
a)
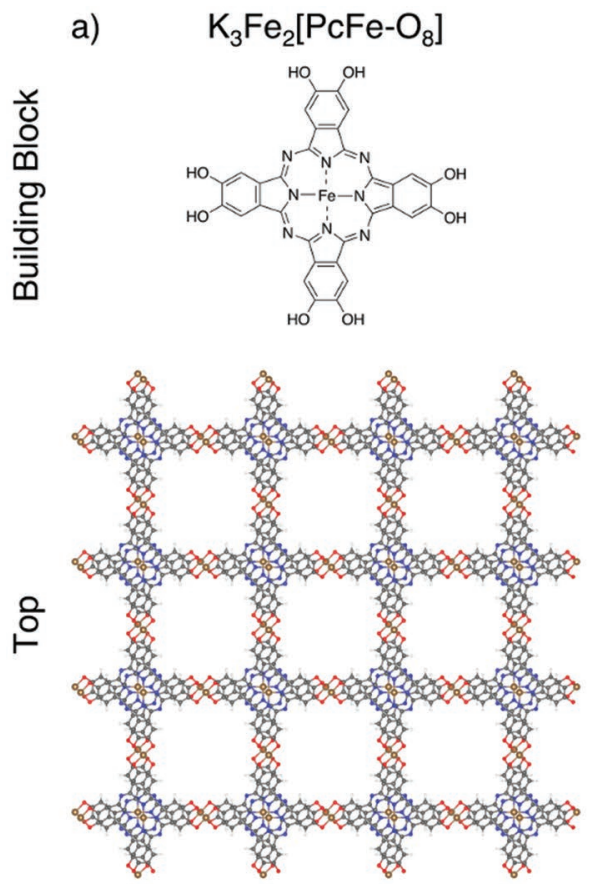

$\frac{0}{0}$ b)
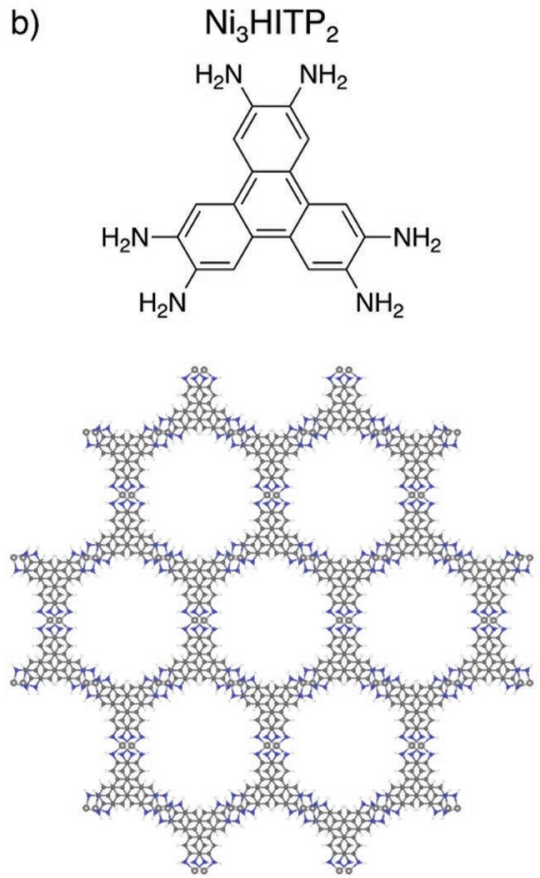

ond c)<smiles>CC(C)(C)[Se]c1c([SeH])c([SeH])c([SeH])c([SeH])c1[SeH]</smiles>

Figure 5. Building blocks together with the top and side views of the atomically thin layered MOFs: a) $\mathrm{K}_{3} \mathrm{Fe}_{2}\left[\mathrm{PcFe}-\mathrm{O}_{8}\right]\left(\mathrm{Pc}\right.$, phthalocyanine), b) Ni ${ }_{3} \mathrm{HITP} \mathrm{P}_{2}$ (HITP, 2,3,6,7,10,11-hexaiminotriphenylene), and $\left[\mathrm{Cu}_{3}\left(\mathrm{C}_{6} \mathrm{Se}_{6}\right)\right]_{n}$. All systems are in $A A^{\prime} A A^{\prime}$ stacking. Pictures of structures made with VESTA.[55]

a less stable system due to unfavorable Coulomb and van der Waals interactions. Computational investigations showed ${ }^{[7]}$ that two other stackings are energetically more favorable. These are slipped and inclined stackings (Figure 6b,c), with P2 or C2 symmetries, respectively, which emerge in bulk synthesis. ${ }^{[110,112,113]}$ However, in the SURMOF approach, the metastable P4 symmetry with eclipsed stacking is enforced by the anchoring of the first MOF layers to the nucleating surface.

We have calculated the corresponding electronic band structures for all three stackings of Cu-SURMOF-2 (Figure 6). While the in-plane bands are nearly dispersionless, the stacking induces dispersion in the out-of-plane directions $(\Gamma$ to $Z$ ). The conduction band is formed by dispersionless single state in all cases, which corresponds to the $\mathrm{Cu} d$-orbitals. On the other hand, the valence band is dominated by $\mathrm{C} p$-orbitals of the organic linkers. The P2 or C2 systems exhibit heavy electrons throughout the Brillouin zone, similar as in P4. However, light holes emerge due to stacking-induced band dispersion in the direction perpendicular to the layers.

The first report on indirect band gap formation in MOFs was published in 2015.[43] Thin films of epitaxial MOFs have been studied, and photoinduced charge-carrier generation was observed. The investigated Zn-paddle wheel SURMOF-2 derivative utilized Pd-porphyrinoid linkers (Pd-PP-SURMOF, see Figure 7a). As parent SURMOF-2, this structure is also a square lattice in-plane and layers are stacked in the AAAA fashion. Such a system results in fairly dispersionless bands of the electronic structure (Figure 7b), however, zoom-in to the conduction and valence bands reveals dispersion of a few $\mathrm{meV}$.
This value corresponds to a mobility of about $0.003 \mathrm{~cm}^{2} \mathrm{~V}^{-1} \mathrm{~s}^{-1}$, which at that time was larger than for any other MOF. This MOF exhibits an indirect band gap, which should result in suppressed electron-hole recombination and improved photovoltaic properties in such organic-semiconductor-based devices.

The photovoltaic efficiency of the reported PP-SURMOF amounts to only $0.2 \%,{ }^{[43]}$ and is thus far too low for realizing a competitive device. Another issue is the absorbance of the PP itself: The strongly absorbing Soret band is in the ultraviolet, while the $\mathrm{Q}$ bands, which are located in the visible spectrum, are only weakly absorbing. PP functionalization can strongly enhance the absorbance of the $\mathrm{Q}$ bands. Among the large number of possibilities, three particularly interesting ones have been identified by combining rational design with computational screening. ${ }^{[42]} 1$ ) Distorting the planarity alters the selection rules and, thus, enhances the intensity of the $\mathrm{Q}$ bands. This can be achieved by bromination of the PP core (twisted octabromo porphyrin). 2) The $\pi$-conjugation of the PP can be extended by adding a phenyl-acetylene (PA) group. 3) The $\pi$-system can be affected by the presence of electron-withdrawing fluorinated phenyl substituents. ${ }^{[42]}$ All three strategies show an effect on the isolated PP linker molecules, in particular, the $\mathrm{Q}$ band intensity increases. Incorporated to a PP-SURMOF, these three linkers, however, show very different absorbance. This can, again, be attributed to the proximity effect.

For the brominated linker, the geometrically distorted building blocks fail to arrange themselves into well-ordered stacks. As a result, loosely packed linker stacks with distances of $\approx 6.1 \AA$, too far to cause a significant proximity effect, are 


\section{Cu-SURMOF-2}
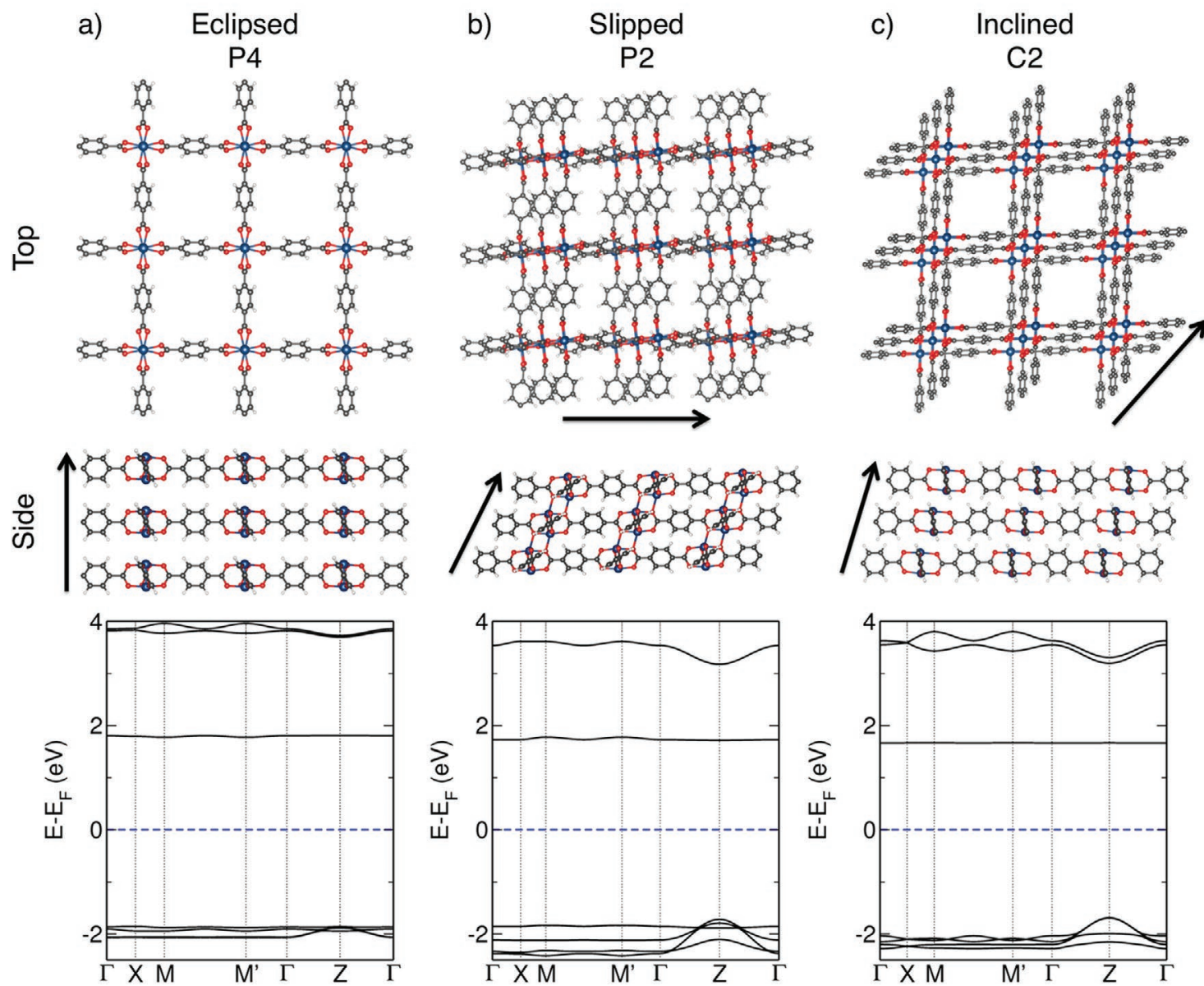

Figure 6. (Top and middle panel) Top and side views of different stackings in SURMOF-2 based on Cu-paddle wheel and 1,4-benzene dicarboxylate linkers. (Bottom panel) corresponding band structures calculated on the optimized structures from ref. [7] at the DFT/PBE0-D3 level of theory with all electron basis set (double zeta with polarization on light elements and triple zeta with polarization for $\mathrm{Cu}$ atoms) as implemented in Crystal14. ${ }^{[144]}$ The $\mathrm{Cu}$ atoms were antiferromagnetically coupled in the present simulations (bands corresponding to $\alpha$ and $\beta$ electrons are identical). Slipped and inclined stacking of SURMOF-2 layers induces out-of-plane dispersion in band structure, due to increased van der Waals and preferable Coulomb interactions. Pictures of structures made with VESTA. ${ }^{[55]}$

obtained. Hence, the incorporation of the brominated PP (1) into the MOF lattice does not affect the absorber properties significantly. For the fluorinated species (3), also the formation of a well-ordered lattice is reported. However, Coulomb repulsion keeps the linkers at the widest possible distance $(\approx 6.3 \AA)$ and the resulting absorption spectrum is very similar to that of the individual molecules. If large and aromatic linkers are used (as in case of PA (2)), the attractive London dispersion interaction fosters linker rotation to the extent that the PP molecules form well-ordered stacks with intermolecular distance of $\approx 3.3 \AA$, very close to that in graphite. Consequently, the band structure shows strong dispersion, which results in a red shift of the Soret band and enhances and broadens the Q bands. These results are summarized in Figure 8 and reported in ref. [42].

To what degree is the spatial extension of the aromatic system of the individual porphyrinic linkers relevant to the properties, which are mainly caused by the proximity effect in the linker stack? To answer this question, we analyzed the band dispersion as function of the rotation angle between the
PP moiety and the PA substituent (Figure 9). This is achieved by rotating parts of the linker (PA and the benzene rings connected to the paddle wheel). Indeed, such a rotation results in a strong manipulation of the electronic bands, from almost flat conduction band in a hypothetical structure with all likers at $0^{\circ}$ with respect to the PP to very strong dispersion in both band edges for rotations of both parts by $\pm 110^{\circ}$. We believe that such rotations can be achieved by proper selection of functional groups (steric control units, SCUs, see below) in the PP linkers.

In the previous paragraphs, we have shown that wellarranged stacks of aromatic molecules can provide additional functionality to MOFs. The precise control of stacking of MOF layers can be tuned by introducing SCUs (see Figure 10). As demonstrated in recent work, when restricting the size of these units to values smaller than the MOF pore size, the packing of aromatic moieties within the MOF linkers can be varied without changing overall lattice parameters of the MOF material. ${ }^{[115]}$ In particular, by first creating libraries in silico using appropriate computational workflows (as described in refs. [115,116]), 


\section{a) \\ Pd-porphyrin Zn-SURMOF}

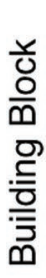

HOOC

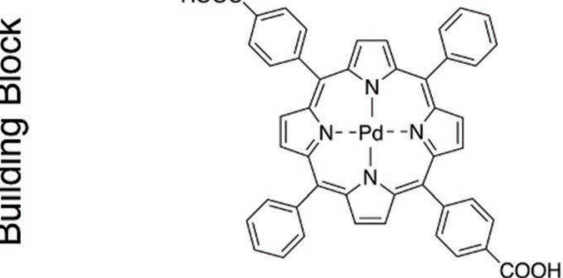

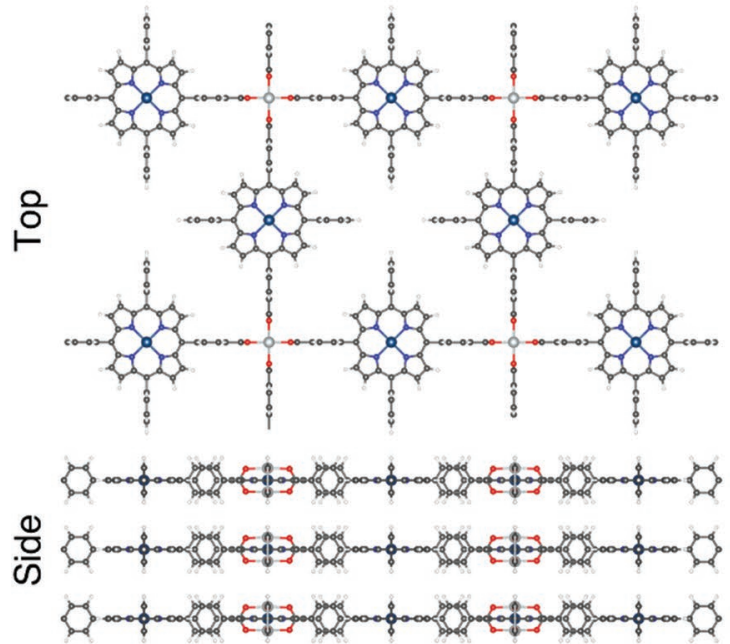

b)

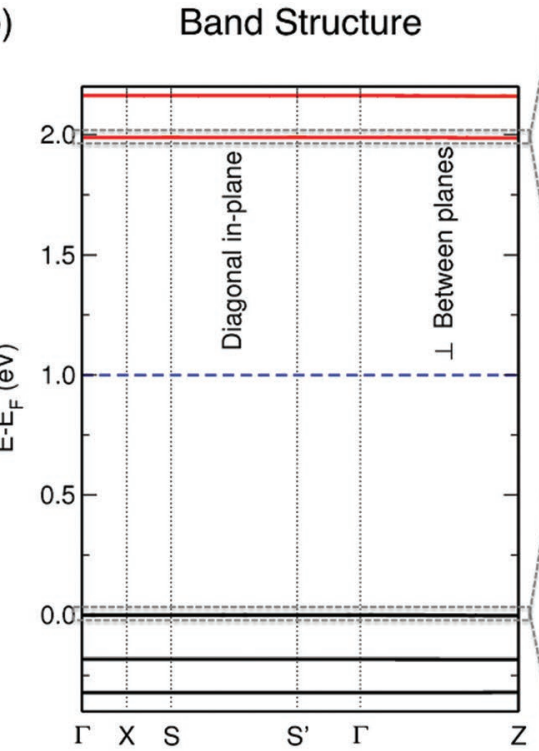

Figure 7. a) Building block (15-diphenyl-10, 20-di(4-carboxyphenyl)porphyrin, Pd-porphyrin) together with the top and side views of Pd-porphyrinbased Zn-SURMOF, and b) the corresponding band structure with zoom-in to the top of valence and bottom of conduction bands. Adapted with permission. ${ }^{[43]}$ Copyright 2015, Wiley. Bands are fairly flat, however, small out-of-plane dispersion occurs in the direction perpendicular to the layers. The dispersion is in the limit of a couple of meV. Pictures of structures made with VESTA. ${ }^{[55]}$

the structures of interest can be identified by computational screening of large libraries, before linkers equipped with the "best" SCU are synthesized and used to assemble the corresponding MOFs or SURMOFs.

Additionally, the individual MOF layers provide strong anisotropic transport properties for excitons and other optical excitations. ${ }^{[117]}$ In some systems, exceptionally large exciton diffusion lengths have been determined experimentally. ${ }^{[118]}$ In that work, it was proposed that the observed experimental value of around $100 \mathrm{~nm}$ is actually only limited by the domain size of the investigated SURMOFs. Based on theoretical considerations, the intrinsic exciton diffusion length should reach the micrometerregime, which would make this thin layer MOF one of the best exciton transport materials.

\section{Conclusion}

This Progress Report shows that, in addition to molecular functional groups, undercoordinated metal sites, porosity, and large surface areas, a further possibility of property control can be incorporated into crystalline framework materials, such as COFs and MOFs: If aromatic molecules are placed in well-controlled stacks, the proximity effect gives raise to strong electronic effects. If the intermolecular distance between the basal planes of the aromatic molecules is in the range of the interlayer distance of graphene $(\approx 3-3.5 \AA)$, disperse electronic bands emerge, resulting in a ballistic charge carrier transport with appreciable mobilities. Thus, while the electronic properties of most framework materials are merely the superposition of the electronic properties of the constituting molecular building blocks, a suitable stacking of aromatic building blocks can turn them into semiconducting materials with particular electronic and optoelectronic properties.

Without steric control and sufficient flexibility, van der Waals interactions result in self-assembly of stacks with strong proximity effect. Mutual shift and twist between the basal planes of the aromatic molecules and intermolecular distance have a strong impact on the resulting electronic structure change, due to the proximity effect. It is possible to control the stacking by strong interlayer interactions, functional groups, or SCUs. These control mechanisms are still beyond the state-of-the-art and subject of the authors' ongoing research efforts.

As recent results of the physics community on twisted bilayer graphene and 2D van der Waals heterostructures demonstrate, many new effects are possible by exploiting the proximity effect. These so-called quantum materials include Mott insulators, superconducting states, Majorana fermions, and topological states. The rich structural and chemical variety of MOFs and COFs opens the door to exploit these effects in materials that emerge from chemistry. It will pave the way to a wealth of opportunities, for example, exploiting topological 
a) $\mathrm{Br}$-Zn-SURMOF

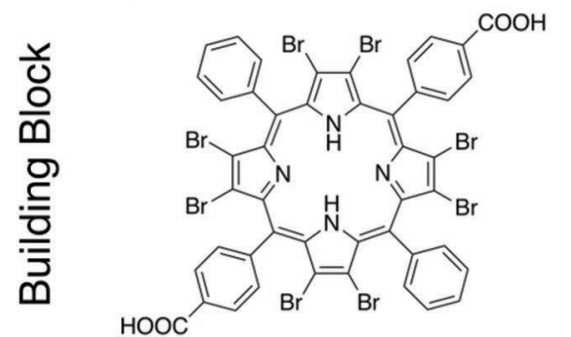

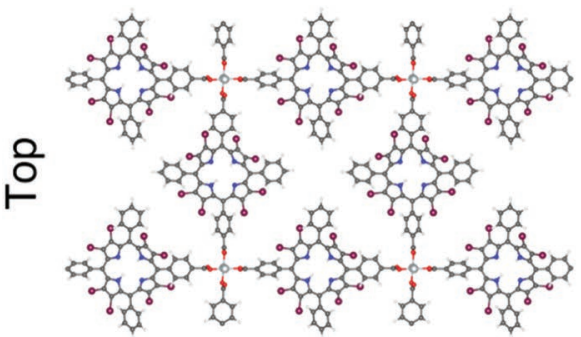
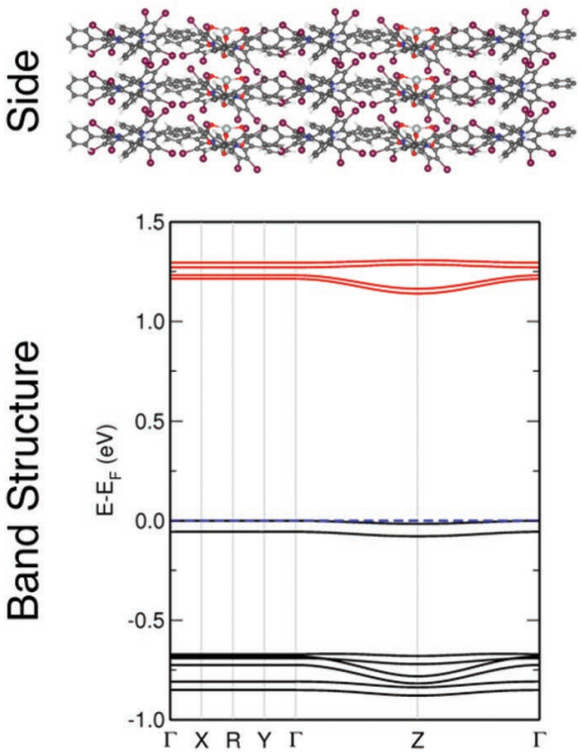

b) PA-Zn-SURMOF<smiles></smiles>

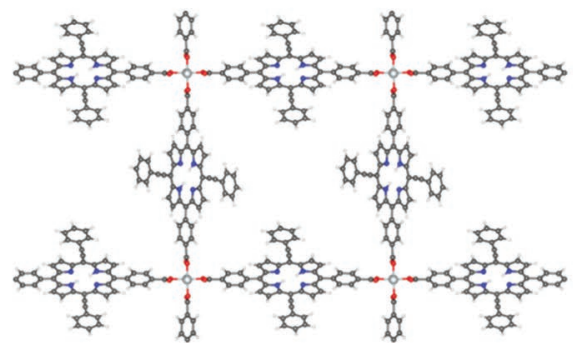
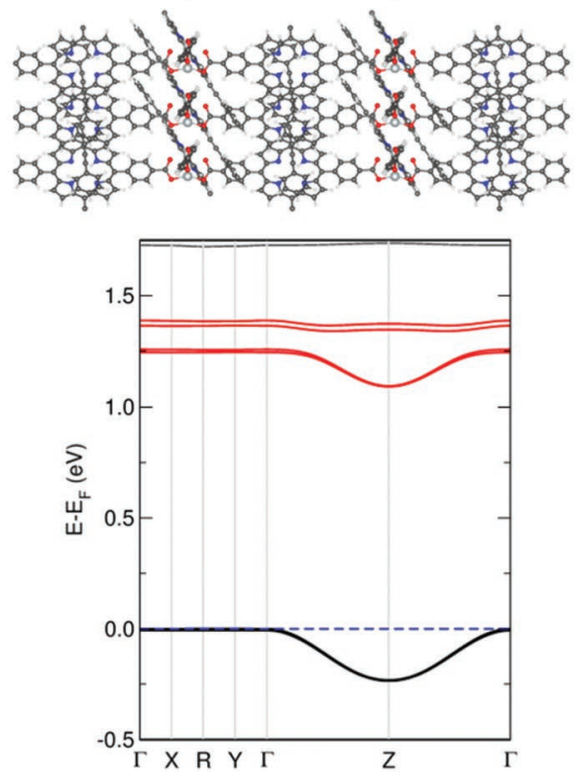

c) F-Zn-SURMOF

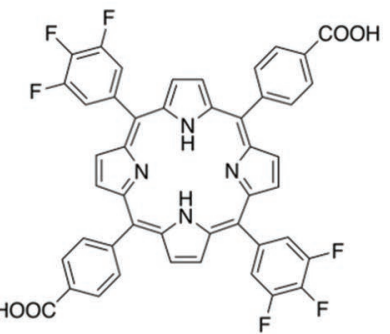

Figure 8. PP-SURMOF-design. (Top panel) Building units together with the top and side views of three Zn-SURMOFs with different PP linkers: a) phenyl linkers and $\mathrm{Br}$ functionalization of PP (Br-Zn-SURMOF), b) phenyl acetylene functional groups (PA-Zn-SURMOF), and c) fluorinated phenyl functional groups (F-Zn-SURMOF). (Bottom panel) The corresponding band structures. Adapted with permission. ${ }^{[42]}$ Copyright 2019, Wiley. Strong band dispersion observed for the PA-Zn-SURMOF in the stacking direction, due to enhanced London dispersion interactions between the PP linkers. Pictures of structures made with VESTA. ${ }^{[55]}$

states to drive chemical reactions as catalysts or to control molecular separation, to form high-density well-ordered molecular wires, and to form interfaces between quantum materials and liquids or nanoparticles.

\section{Experimental Section}

Computational Methods: This section gives an overview of methods that are useful for computationally tackling layered COFs and MOFs, from constructing the atomistic structures to obtain high-level electronic structure data. It includes the methods that have been used to obtain the results discussed above. The purpose is to allow newcomers to quickly enter the exciting field of framework materials.
Structure Builder: Many MOFs and COFs have non-trivial geometry and are composed of rather large, sometimes bulky, molecules. Several methods have been developed to stitch these building blocks together to form a framework, including AuToGraFS, ${ }^{[116]}$ zeo++, ${ }^{[119]}$ and MOFplus. ${ }^{[120]}$ Coupling framework generation to a force field is essential to produce starting structures that should be followed by higher-level methods. In this work, the universal force field (UFF) ${ }^{[121]}$ extended for MOFs ${ }^{[122,123]}$ and hydrogen bonds were employed. ${ }^{[124]}$ There are various other forcefield alternatives available for MOFs, which typically perform superior for a particular MOF subclass, but do not provide the almost complete range of elements that are covered by extended UFF. Some ${ }^{[125]}$ can, however, produce superior structures that are comparable to those of quantum chemical approaches. For an overview on alternatives, a recent review by Boyd et al. ${ }^{[126]}$ is referred to. For COFs, incorporating only first and second row atoms, a large variety of standard force fields are available. 
a)

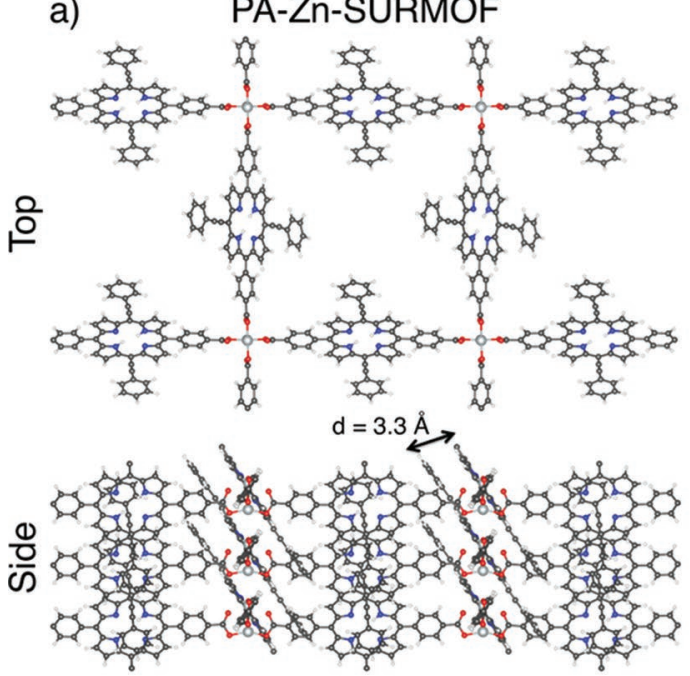

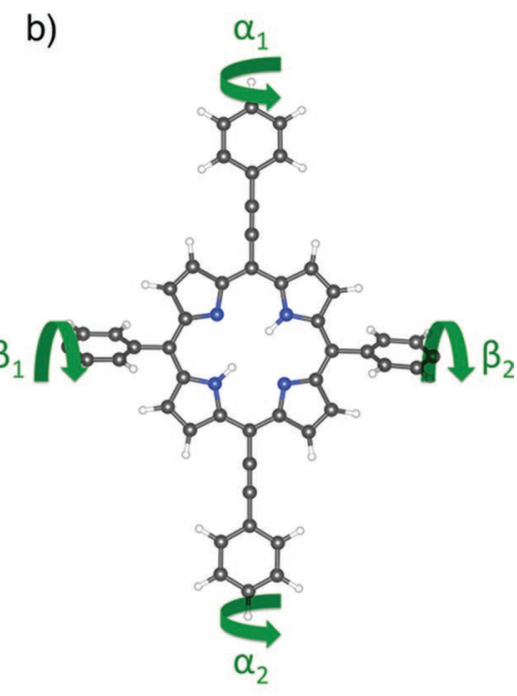

c) $\alpha_{1,2}=0^{\circ}$

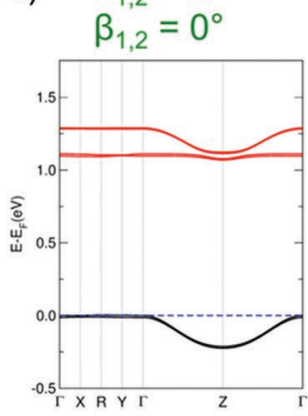

$\alpha_{1,2}=0^{\circ}$

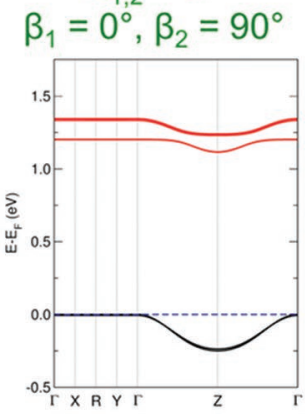

$\alpha_{1,2}=0^{\circ}$ $\beta_{1,2}=110^{\circ}$

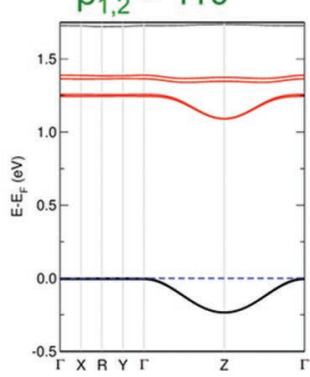

$\alpha_{1,2}=0^{\circ}$

$\beta_{1}=110^{\circ}, \beta_{2}=-110^{\circ}$

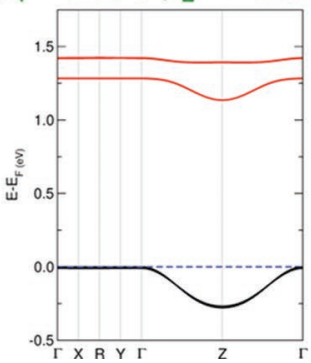

$\alpha_{1,2}=0^{\circ}$

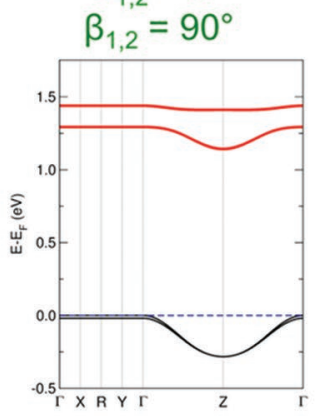

$\alpha_{1}=110^{\circ}, \alpha_{2}=-110^{\circ}$

$\beta_{1}=110^{\circ}, \beta_{2}=-110^{\circ}$

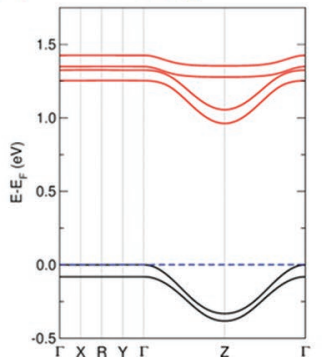

Figure 9. a) Top and side view of the PA-Zn-SURMOF (PA, phenyl acetylene functional group). The interlayer distance $(d=3.3 \AA$ ) corresponds to the distance between PP units in adjacent layers and is close to that in graphite. b) Cluster structure of the linker with possible rotations angles, $\alpha_{\mathrm{i}}$ and $\beta_{\mathrm{i}}$, of the functional groups with respect to the PP plane. c) Band structures corresponding to different values of $\alpha_{\mathrm{i}}$ and $\beta_{\mathrm{i}}$. The strongest band dispersion was obtained for both angles of about $\pm 110^{\circ}$. The band structure from ref. [42] corresponds to the case of $\alpha_{\mathrm{i}}=0^{\circ}$ and $\beta_{\mathrm{i}}=110^{\circ}$. Calculations at the same level of theory as in ref. [42]. Pictures of structures made with VESTA.[55]

DFTB: The DFTB method ${ }^{[127,128]}$ is an approximation to densityfunctional theory (DFT). It employs a minimal atomic-orbital valence basis and involves two-center approximations to the Kohn-Sham matrix elements, thus, allowing the Slater-Koster technique for precalculation of all integrals, which is the key ingredient to allow a performance boost of about three orders of magnitude with respect to DFT. It is available in three variants, the original DFTB form, DFTB0, does not correct for interatomic charge transfer. The self-consistent charge (SCC) approximation, SCC-DFTB, ${ }^{[129]}$ also known as DFTB2, improves the transferability, in particular to polar systems. It has been later improved to the third-order level (DFTB3). ${ }^{[130]}$ Most widely applied parameters include the mat-sci parameters for DFTB0, ${ }^{[131]}$ the mio parameters for SCC-DFTB, and more recently the $3 \mathrm{ob}^{[132]}$ parametrization, applicable to DFTB2 and DFTB3. Spin polarization ${ }^{[133,134]}$ and spin-orbit coupling $(\mathrm{SOC})^{[135]}$ can also be included. London dispersion interactions are commonly treated using an a posteriori correction ${ }^{[136,137]}$ or the manybody dispersion (MBD) approach. ${ }^{[138]}$ DFTB2 has been successfully applied to 2D COFs, ${ }^{[27,59,78,139-152]}$ DFTB3 has not yet been tested carefully with SOC and with periodic boundary conditions. Popular codes covering MOFs and COFs in periodic boundary conditions are $\mathrm{dftb}+{ }^{[153]}$ and AMS/DFTB. ${ }^{[154]}$ The method allows to determine the structure, stacking, electronic, including topological, properties in a 


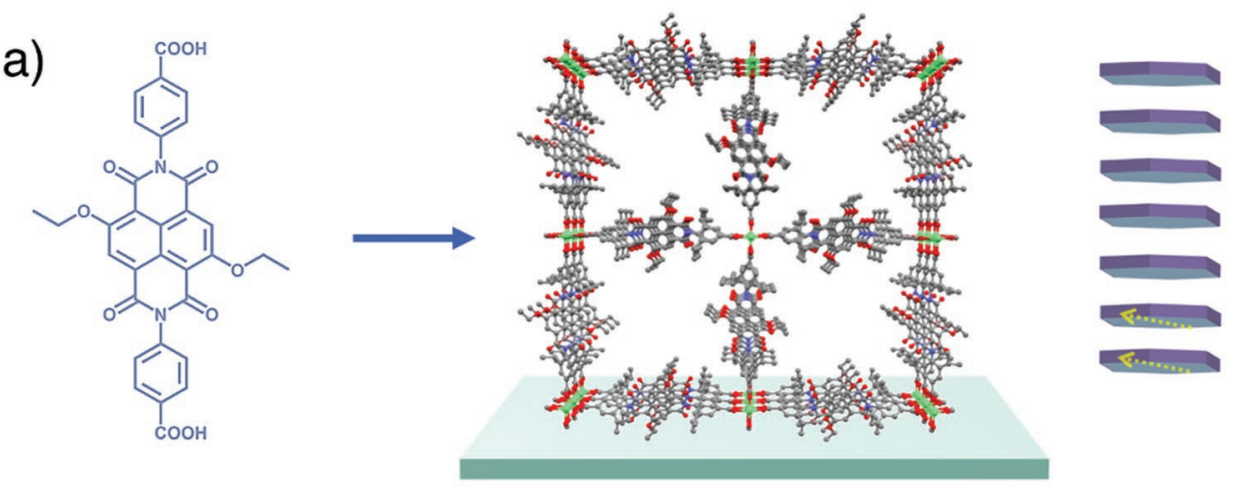

b)

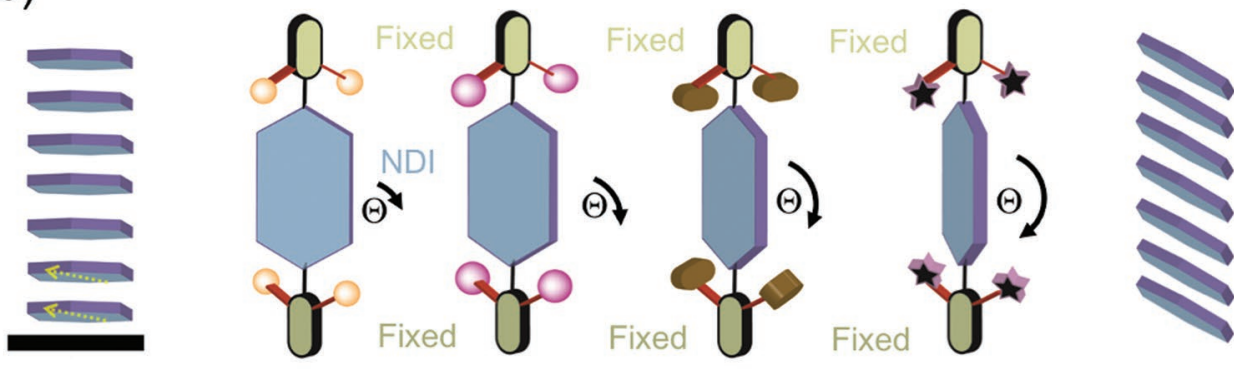

C)

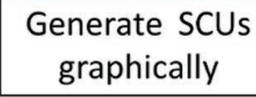

Generate SCUs graphically

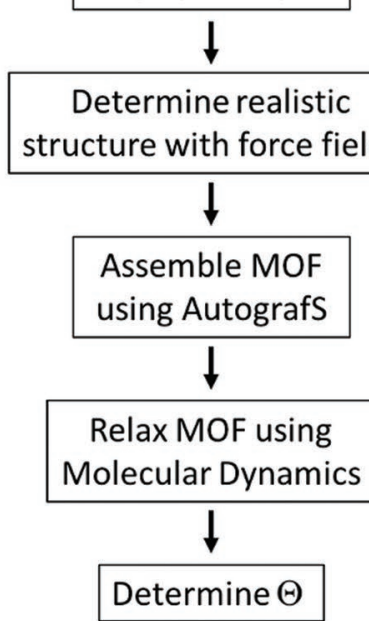

d)

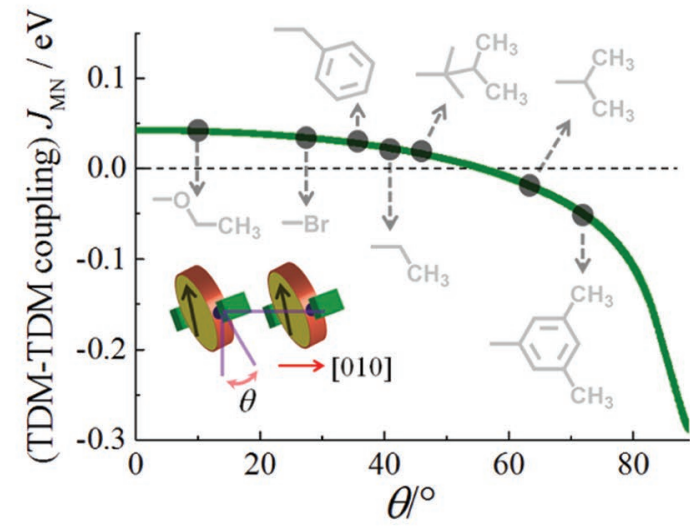

Crystal Engineering:

Reducing the problem from 3D to (essentially) 1D

Figure 10. Employing crystal engineering to tune excitonic coupling in chromophoric SURMOFs using steric control units (SCUs). a) Without SCUs, the naphthalene-diimide (NDI) linkers yield a non-luminescent assembly ( $\mathrm{H}$-aggregate). A theoretical analysis revealed that increasing the angle Q controlling the stacking of the linkers b) modifies the coupling such that luminescence is recovered. c) The workflow used to screen the library of possible linkers is indicated in the lower left. d) TDM-TDM coupling changes sign (TDM, transition dipole moment). Adapted with permission. ${ }^{[15]}$ Copyright 2019, Springer Nature.

first screening approach. An interesting approach to tackle large MOFs and COFs without interlayer conjugation is the fragmented molecular orbitals approach (FMO-DFTB) developed in the Irle group and available in Gamess-US.[155] For more elaborate investigations, DFTB should be substantiated by DFT.

DFT for Periodic Systems: DFT has become the working horse for the calculations of structures and properties of MOFs and COFs. Unfortunately, there is no DFT software available that simultaneously offers all necessary features for this materials class. Periodic systems are typically efficiently treated using plane wave basis sets in conjunction with the projected augmented wave (PAW) method. ${ }^{[156]}$ They are readily available in commercial (VASP, [157] CASTEP[158]) and free (QuantumEspresso ${ }^{[159]}$ ) software. Large pores and the possibility to directly compare to molecular systems makes also local basis function approaches interesting, with typical representatives being

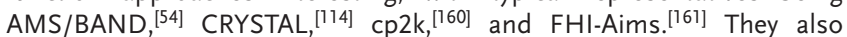
offer access to computationally affordable hybrid functionals PBE0[162] and HSE06. ${ }^{[163]}$ London dispersion interactions need to be accounted for, most popular is the D3 approach by Grimme ${ }^{[164]}$ or the MBD approach. ${ }^{[165]}$ Local basis functions should have triple-zeta quality with 


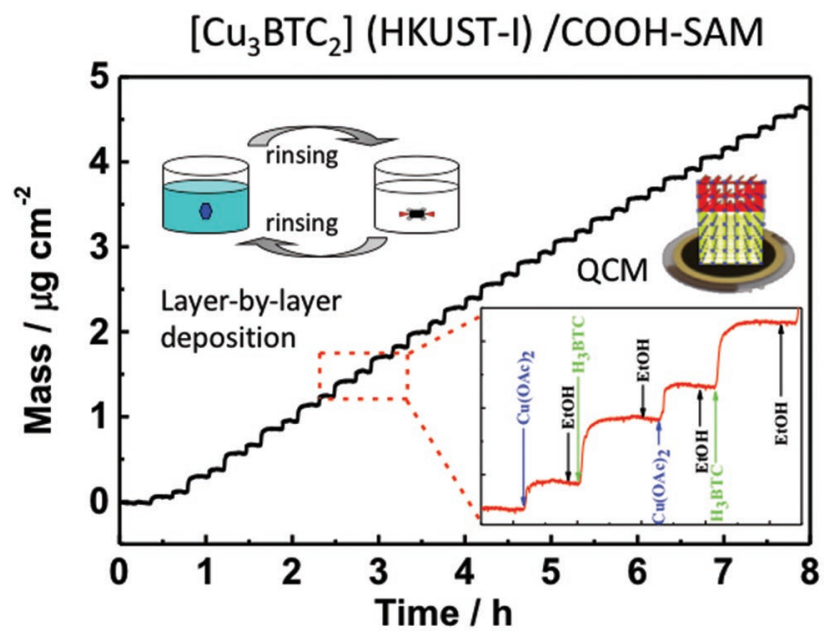

Figure 11. Layer-by-layer growth of a HKUST-I SURMOF on a quartzcrystal microbalance (QCM) crystal. From the frequency shift of the QCM substrate, the deposited mass can be determined in a quantitative fashion. Note that the growth is self-terminating, for example, for the metal source the increase of mass saturates after a few minutes. Data are taken from ref. [172].

polarization functions to allow for the correct description of the decay of the electron density into the pore centers.

For geometry optimizations and low-level band structure calculations, a gradient-corrected local functional is sufficient, most prominently the PBE functional is employed, ${ }^{[166]}$ while band gaps should be improved using a hybrid approach, such as PBE0 ${ }^{[162]}$ or HSE06. ${ }^{[163]}$ For heavier nuclei, scalar relativistic effects can be treated either using the zeroorder regular approximation (ZORA), ${ }^{[167]}$ relativistically corrected pseudopotentials or effective-core potentials. DFT allows also the calculation of spin-spin couplings, which are necessary to account for magnetic ordering. In very demanding cases, wave-function-based methods, such as complete active space self-consistent field (CASSCF) calculations, have been employed to accurately describe excited states. ${ }^{[23]}$

Beyond DFT: There are well-known limitations for electronic structure calculations using DFT, most notably the too-narrow band gaps. A thorough validation of using methods beyond DFT is, therefore, desirable, even though often impossible due to the computational costs. Options include the quasi-particle model GLLB-SC, ${ }^{[168,169]}$ which is available in AMS/BAND, and GW and RPA methods, ${ }^{[170]}$ as provided in FHI-Aims and VASP.

Layer-by-Layer Growth Technique: While the bulk powder form of MOFs is only of limited interest with regard to the fabrication of stacked layers, the Ibl or quasi-epitaxial growth of SURMOFs offers a number of interesting options. Briefly, the $\mathrm{lbl}$ process proceeds by repeating a four-step cycle comprising the sequential immersion into the two solutions of the individual reactants, separated by rinsing with a solvent (typically ethanol) (Figure 11). Thus, instead of mixing the reactants like in the conventional solvothermal bulk MOF synthesis, the metal or metal/oxo source is kept apart from the linker solution. Another important difference is that the $\mid \mathrm{bl}$ synthesis is carried out at temperatures much lower than these used in the solvothermal synthesis, in some cases growth already occurs at room temperature. To nucleate the growth of SURMOFs on the substrate, the exposed surface first needs to be functionalized with specific functional groups, thus, yielding $-\mathrm{COOH},-\mathrm{OH}$, or pyridyl terminations. ${ }^{[171]}$ The first step typically is an exposure to the metal source (e.g., $\mathrm{Cu}-$ or $\mathrm{Zn}$-acetate). Subsequently, the other steps (rinsing with ethanol, exposure to organic linker, rinsing with ethanol) are repeated until the desired thickness is reached. After synthesis, the SURMOF structure can be rigorously characterized by out-of-plane and in-plane X-ray diffraction. ${ }^{[72]}$

With regard to the topic of this review, the fabrication of stacked layers, it is important to note that SURMOF heterolayers, that is, stacked
2D crystalline coordination networks, can be realized in a straightforward fashion by switching to different linkers and/or nodes during the $\mid \mathrm{bl}$ process. Thus, a programmed assembly of arbitrary sequences of MOF heterolayers becomes possible. ${ }^{[173]}$ While in most cases stacked heterolayers are achieved by switching the organic linkers, in a few cases, also the metal nodes have been varied, for example, in case of $\mathrm{Cu} / \mathrm{Zn}{ }^{[174]}$ Particularly interesting, with regard to realizing mixed-metal MOFs, are lanthanide-based SURMOFs, since the very similar coordination chemistry of these metal ions allows switching between the different elements without pronounced changes of the MOF structure. ${ }^{[175]}$

\section{Acknowledgements}

K.B., T.H., and C.W. thank Deutsche Forschungsgemeinschaft within the Priority Program COORNETs (SPP 1928). C.W. acknowledges support through the Cluster "3DMM2O" funded by Deutsche Forschungsgemeinschaft. A.K., M.A.S., K.B., and T.H. thank ZIH Dresden for computational resources.

\section{Conflict of Interest}

The authors declare no conflict of interest.

\section{Keywords}

covalent organic frameworks, electronic structure, layered materials, metal-organic frameworks, proximity effect

Received: September 27, 2019

Revised: November 9, 2019

Published online: February 6, 2020

[1] H. Li, M. Eddaoudi, M. O'Keeffe, O. M. Yaghi, Nature 1999, 402, 276.

[2] J. L. C. Rowsell, O. M. Yaghi, Microporous Mesoporous Mater. 2004, $73,3$.

[3] J. R. Long, O. M. Yaghi, Chem. Soc. Rev. 2009, 38, 1213.

[4] S. R. Batten, S. M. Neville, D. R. Turner, Coordination Polymers, The Royal Society of Chemistry, Washington, DC 2009.

[5] A. P. Côté, A. I. Benin, N. W. Ockwig, M. O'Keeffe, A. J. Matzger, O. M. Yaghi, Science 2005, 310, 1166.

[6] C. S. Diercks, O. M. Yaghi, Science 2017, 355, eaal1585.

[7] J. Liu, B. Lukose, O. Shekhah, H. K. Arslan, P. Weidler, H. Gliemann, S. Bräse, S. Grosjean, A. Godt, X. Feng, K. Müllen, I.-B. Magdau, T. Heine, C. Wöll, Sci. Rep. 2012, 2, 921.

[8] H. Deng, S. Grunder, K. E. Cordova, C. Valente, H. Furukawa, M. Hmadeh, F. Gandara, A. C. Whalley, Z. Liu, S. Asahina, H. Kazumori, M. O'Keeffe, O. Terasaki, J. F. Stoddart, O. M. Yaghi, Science 2012, 336, 1018

[9] I. M. Hönicke, I. Senkovska, V. Bon, I. A. Baburin, N. Bönisch, S. Raschke, J. D. Evans, S. Kaskel, Angew. Chem., Int. Ed. 2018, 57, 13780.

[10] H. Furukawa, N. Ko, Y. B. Go, N. Aratani, S. B. Choi, E. Choi, A. O. Yazaydin, R. Q. Snurr, M. O'Keeffe, J. Kim, O. M. Yaghi, Science 2010, 329, 424.

[11] M. P. Suh, H. J. Park, T. K. Prasad, D.-W. Lim, Chem. Rev. 2012, 112, 782.

[12] M. Wahiduzzaman, C. F. J. Walther, T. Heine, J. Chem. Phys. 2014, $141,064708$.

[13] Z. Hu, Y. Wang, B. B. Shah, D. Zhao, Adv. Sustainable Syst. 2019, 3,1800080 . 
[14] M. Ding, R. W. Flaig, H.-L. Jiang, O. M. Yaghi, Chem. Soc. Rev. 2019, 48, 2783

[15] J. Y. Kim, H. Oh, H. R. Moon, Adv. Mater. 2019, 31, 1805293.

[16] I. Weinrauch, I. Savchenko, D. Denysenko, S. M. Souliou, H.-H. Kim, M. Le Tacon, L. L. Daemen, Y. Cheng, A. Mavrandonakis, A. J. Ramirez-Cuesta, D. Volkmer, G. Schütz, M. Hirscher, T. Heine, Nat. Commun. 2017, 8, 14496.

[17] Z. Wang, A. Knebel, S. Grosjean, D. Wagner, S. Bräse, C. Wöll, J. Caro, L. Heinke, Nat. Commun. 2016, 7, 13872.

[18] A. Knebel, B. Geppert, K. Volgmann, D. I. Kolokolov, A. G. Stepanov, J. Twiefel, P. Heitjans, D. Volkmer, J. Caro, Science 2017, 358, 347.

[19] C. J. Doonan, C. J. Sumby, CrystEngComm 2017, 19, 4044.

[20] S. R. Batten, N. R. Champness, X.-M. Chen, J. Garcia-Martinez, S. Kitagawa, L. Öhrström, M. O'Keeffe, M. Paik Suh, J. Reedijk, Pure Appl. Chem. 2013, 85, 1715

[21] R. Hoffmann, Solids and Surfaces: A Chemist's View of Bonding in Extended Structures (Print on Demand Edition 1988), Wiley-VCH, New York 2002.

[22] P. St. Petkov, G. N. Vayssilov, J. Liu, O. Shekhah, Y. Wang, C. Wöll, T. Heine, ChemPhysChem 2012, 13, 2025.

[23] K. Müller, K. Fink, L. Schöttner, M. Koenig, L. Heinke, C. Wöll, ACS Appl. Mater. Interfaces 2017, 9, 37463.

[24] C. Yang, R. Dong, M. Wang, P. S. Petkov, Z. Zhang, M. Wang, P. Han, M. Ballabio, S. A. Bräuninger, Z. Liao, J. Zhang, F. Schwotzer, E. Zschech, H.-H. Klauss, E. Cánovas, S. Kaskel, M. Bonn, S. Zhou, T. Heine, X. Feng, Nat. Commun. 2019, 10, 3260.

[25] S. Friedlaender, J. Liu, M. Addicoat, P. Petkov, N. Vankova, R. Rueger, A. Kuc, W. Guo, W. Zhou, B. Lukose, Z. Wang, P. G. Weidler, A. Poeppl, M. Ziese, T. Heine, C. Woell, Angew. Chem., Int. Ed. 2016, 55, 12683.

[26] M. Kurmoo, Chem. Soc. Rev. 2009, 38, 1353.

[27] E. Jin, M. Asada, Q. Xu, S. Dalapati, M. A. Addicoat, M. A. Brady, H. Xu, T. Nakamura, T. Heine, Q. Chen, D. Jiang, Science 2017, 357, 673.

[28] R. Dong, P. Han, H. Arora, M. Ballabio, M. Karakus, Z. Zhang, C. Shekhar, P. Adler, P. S. Petkov, A. Erbe, S. C. B. Mannsfeld, C. Felser, T. Heine, M. Bonn, X. Feng, E. Cánovas, Nat. Mater. 2018, 17, 1027.

[29] R. Dong, Z. Zhang, D. C. Tranca, S. Zhou, M. Wang, P. Adler, Z. Liao, F. Liu, Y. Sun, W. Shi, Z. Zhang, E. Zschech, S. C. B. Mannsfeld, C. Felser, X. Feng, Nat. Commun. 2018, 9, 2637.

[30] X. Zhuang, W. Zhao, F. Zhang, Y. Cao, F. Liu, S. Bi, X. Feng, Polym. Chem. 2016, 7, 4176 .

[31] Y. Cao, V. Fatemi, S. Fang, K. Watanabe, T. Taniguchi, E. Kaxiras, P. Jarillo-Herrero, Nature 2018, 556, 43.

[32] D. R. Klein, D. MacNeill, J. L. Lado, D. Soriano, E. NavarroMoratalla, K. Watanabe, T. Taniguchi, S. Manni, P. Canfield, J. Fernández-Rossier, P. Jarillo-Herrero, Science 2018, 360, 1218.

[33] R. Ribeiro-Palau, C. Zhang, K. Watanabe, T. Taniguchi, J. Hone, C. R. Dean, Science 2018, 361, 690.

[34] K. K. Kim, H. S. Lee, Y. H. Lee, Chem. Soc. Rev. 2018, 47, 6342.

[35] D. L. Duong, S. J. Yun, Y. H. Lee, ACS Nano 2017, 11, 11803.

[36] R. Frisenda, E. Navarro-Moratalla, P. Gant, D. Pérez De Lara, P. Jarillo-Herrero, R. V. Gorbachev, A. Castellanos-Gomez, Chem. Soc. Rev. 2018, 47, 53.

[37] S. Carr, D. Massatt, S. Fang, P. Cazeaux, M. Luskin, E. Kaxiras, Phys. Rev. B 2017, 95, 075420.

[38] B. Hunt, J. D. Sanchez-Yamagishi, A. F. Young, M. Yankowitz, B. J. LeRoy, K. Watanabe, T. Taniguchi, P. Moon, M. Koshino, P. Jarillo-Herrero, R. C. Ashoori, Science 2013, 340, 1427.

[39] C. R. Dean, L. Wang, P. Maher, C. Forsythe, F. Ghahari, Y. Gao, J. Katoch, M. Ishigami, P. Moon, M. Koshino, T. Taniguchi, K. Watanabe, K. L. Shepard, J. Hone, P. Kim, Nature 2013, 497, 598.
[40] K. Kim, A. DaSilva, S. Huang, B. Fallahazad, S. Larentis, T. Taniguchi, K. Watanabe, B. J. LeRoy, A. H. MacDonald, E. Tutuc, Proc. Natl. Acad. Sci. U. S. A. 2017, 114, 3364.

[41] X. Ding, L. Chen, Y. Honsho, X. Feng, O. Saengsawang, J. Guo, A. Saeki, S. Seki, S. Irle, S. Nagase, V. Parasuk, D. Jiang, J. Am. Chem. Soc. 2011, 133, 14510.

[42] R. Haldar, K. Batra, S. M. Marschner, A. B. Kuc, S. Zahn, R. A. Fischer, S. Bräse, T. Heine, C. Wöll, Chem. - Eur. J. 2019, 25, 7847.

[43] J. Liu, W. Zhou, J. Liu, I. Howard, G. Kilibarda, S. Schlabach, D. Coupry, M. Addicoat, S. Yoneda, Y. Tsutsui, T. Sakurai, S. Seki, Z. Wang, P. Lindemann, E. Redel, T. Heine, C. Wöll, Angew. Chem., Int. Ed. 2015, 54, 7441.

[44] O. Shekhah, J. Liu, R. A. Fischer, C. Woll, Chem. Soc. Rev. 2011, 40, 1081.

[45] L. Heinke, C. Wöll, Adv. Mater. 2019, 31, 1806324.

[46] K. S. Novoselov, A. K. Geim, S. V. Morozov, D. Jiang, M. I. Katsnelson, I. V. Grigorieva, S. V. Dubonos, A. A. Firsov, Nature 2005, 438, 197.

[47] A. K. Geim, K. S. Novoselov, Nat. Mater. 2007, 6, 183.

[48] C. L. Kane, E. J. Mele, Phys. Rev. Lett. 2005, 95, 226801.

[49] J. D. Bernal, Proc. R. Soc. A 1924, 106, 749.

[50] K. S. Novoselov, E. McCann, S. V. Morozov, V. I. Fal'ko, M. I. Katsnelson, U. Zeitler, D. Jiang, F. Schedin, A. K. Geim, Nat. Phys. 2006, 2, 177.

[51] E. McCann, V. I. Fal'ko, Phys. Rev. Lett. 2006, 96, 086805.

[52] A. Grüneis, C. Attaccalite, L. Wirtz, H. Shiozawa, R. Saito, T. Pichler, A. Rubio, Phys. Rev. B 2008, 78, 205425.

[53] Y. Yao, F. Ye, X.-L. Qi, S.-C. Zhang, Z. Fang, Phys. Rev. B 2007, 75, 041401.

[54] P. H. T. Philipsen, G. te Velde, E. J. Baerends, J. A. Berger, P. L. de Boeij, M. Franchini, J. A. Groeneveld, E. S. Kadantsev, R. Klooster, F. Kootstra, P. Romaniello, M. Raupach, D. G. Skachkov, J. G. Snijders, C. J. O. Verzijl, J. A. Celis Gil, J. M. Thijssen, G. Wiesenekker, C. A. Peeples, G. Schreckenbach, T. Ziegler, BAND2019: SCM, Theoretical Chemistry, Vrije Universiteit, Amsterdam, The Netherlands

[55] K. Momma, F. Izumi, J. Appl. Crystallogr. 2011, 44, 1272.

[56] Y. Cao, V. Fatemi, A. Demir, S. Fang, S. L. Tomarken, J. Y. Luo, J. D. Sanchez-Yamagishi, K. Watanabe, T. Taniguchi, E. Kaxiras, R. C. Ashoori, P. Jarillo-Herrero, Nature 2018, 556, 80.

[57] R. Bistritzer, A. H. MacDonald, Proc. Natl. Acad. Sci. U. S. A. 2011, $108,12233$.

[58] X. Feng, L. Chen, Y. Dong, D. Jiang, Chem. Commun. 2011, 47, 1979.

[59] S. Kandambeth, D. B. Shinde, M. K. Panda, B. Lukose, T. Heine, R. Banerjee, Angew. Chem., Int. Ed. 2013, 52, 13052.

[60] S. Thomas, H. Li, C. Zhong, M. Matsumoto, W. R. Dichtel, J.-L. Bredas, Chem. Mater. 2019, 31, 3051.

[61] M. S. Lohse, T. Bein, Adv. Funct. Mater. 2018, 28, 1705553.

[62] Y. Song, Q. Sun, B. Aguila, S. Ma, Adv. Sci. 2019, 6, 1970011.

[63] B. Lukose, A. Kuc, T. Heine, Chem. - Eur. J. 2011, 17, 2388.

[64] B. Lukose, A. Kuc, J. Frenzel, T. Heine, Beilstein J. Nanotechnol. 2010, 1, 60.

[65] W. Zhou, H. Wu, T. Yildirim, Chem. Phys. Lett. 2010, 499, 103.

[66] Y. Fan, Q. Wen, T.-G. Zhan, Q.-Y. Qi, J.-Q. Xu, X. Zhao, Chem. - Eur. J. 2017, 23, 5668.

[67] X. Liu, J. Tan, A. Wang, X. Zhang, M. Zhao, Phys. Chem. Chem. Phys. 2014, 16, 23286.

[68] S. B. Alahakoon, C. M. Thompson, A. X. Nguyen, G. Occhialini, G. T. McCandless, R. A. Smaldone, Chem. Commun. 2016, 52, 2843.

[69] I. Hisaki, S. Nakagawa, N. Ikenaka, Y. Imamura, M. Katouda, M. Tashiro, H. Tsuchida, T. Ogoshi, H. Sato, N. Tohnai, M. Miyata, J. Am. Chem. Soc. 2016, 138, 6617. 
[70] T.-Y. Zhou, S.-Q. Xu, Q. Wen, Z.-F. Pang, X. Zhao, J. Am. Chem. Soc. 2014, 136, 15885.

[71] Z.-F. Pang, T.-Y. Zhou, R.-R. Liang, Q.-Y. Qi, X. Zhao, Chem. Sci. 2017, 8, 3866.

[72] Y. Tian, S.-Q. Xu, R.-R. Liang, C. Qian, G.-F. Jiang, X. Zhao, CrystEngComm 2017, 19, 4877.

[73] Y. Jing, T. Heine, J. Am. Chem. Soc. 2019, 141, 743.

[74] X. Feng, X. Ding, D. Jiang, Chem. Soc. Rev. 2012, 41, 6010.

[75] R. Dong, T. Zhang, X. Feng, Chem. Rev. 2018, 118, 6189.

[76] H. Wang, Z. Zeng, P. Xu, L. Li, G. Zeng, R. Xiao, Z. Tang, D. Huang, L. Tang, C. Lai, D. Jiang, Y. Liu, H. Yi, L. Qin, S. Ye, X. Ren, W. Tang, Chem. Soc. Rev. 2019, 48, 488.

[77] L. Wang, H. Sahabudeen, T. Zhang, R. Dong, npj 2D Mater. Appl. 2018, 2, 26

[78] N. Huang, L. Zhai, D. E. Coupry, M. A. Addicoat, K. Okushita, K. Nishimura, T. Heine, D. Jiang, Nat. Commun. 2016, 7, 12325.

[79] P. Miró, M. Audiffred, T. Heine, Chem. Soc. Rev. 2014, 43, 6537.

[80] F. Hulliger, F. Lévy, Structural Chemistry of Layer-Type Phases, Springer, Dordrecht, The Netherlands 1977.

[81] S. Haastrup, M. Strange, M. Pandey, T. Deilmann, P. S. Schmidt, N. F. Hinsche, M. N. Gjerding, D. Torelli, P. M. Larsen, A. C. Riis-Jensen, J. Gath, K. W. Jacobsen, J. Jørgen Mortensen, T. Olsen, K. S. Thygesen, 2D Mater. 2018, 5, 042002.

[82] S.-Y. Ding, W. Wang, Chem. Soc. Rev. 2013, 42, 548.

[83] M. Dogru, T. Bein, Chem. Commun. 2014, 50, 5531.

[84] X. Feng, X. Ding, L. Chen, Y. Wu, L. Liu, M. Addicoat, S. Irle, Y. Dong, D. Jiang, Sci. Rep. 2016, 6, 32944.

[85] A. K. Mandal, J. Mahmood, J.-B. Baek, ChemNanoMat 2017, 3, 373.

[86] S. Das, P. Heasman, T. Ben, S. Qiu, Chem. Rev. 2017, 117, 1515.

[87] B. P. Biswal, S. Valligatla, M. Wang, T. Banerjee, N. A. Saad, B. M. K. Mariserla, N. Chandrasekhar, D. Becker, M. Addicoat, I. Senkovska, R. Berger, D. N. Rao, S. Kaskel, X. Feng, Angew. Chem., Int. Ed. 2019, 58, 6896.

[88] D. Er, L. Dong, V. B. Shenoy, J. Phys. Chem. C 2016, 120, 174.

[89] R.-N. Wang, X.-R. Zhang, S.-F. Wang, G.-S. Fu, J.-L. Wang, Phys. Chem. Chem. Phys. 2016, 18, 1258.

[90] S. Thomas, H. Li, R. R. Dasari, A. M. Evans, I. Castano, T. G. Allen, O. G. Reid, G. Rumbles, W. R. Dichtel, N. C. Gianneschi, S. R. Marder, V. Coropceanu, J.-L. Brédas, Mater. Horiz. 2019, 6, 1868.

[91] S. Wan, F. Gándara, A. Asano, H. Furukawa, A. Saeki, S. K. Dey, L. Liao, M. W. Ambrogio, Y. Y. Botros, X. Duan, S. Seki, J. F. Stoddart, O. M. Yaghi, Chem. Mater. 2011, 23, 4094.

[92] J. R. Long, O. M. Yaghi, Chem. Soc. Rev. 2009, 38, 1213.

[93] O. M. Yaghi, M. O'Keeffe, N. W. Ockwig, H. K. Chae, M. Eddaoudi, J. Kim, Nature 2003, 423, 705.

[94] I. Stassen, N. Burtch, A. Talin, P. Falcaro, M. Allendorf, R. Ameloot, Chem. Soc. Rev. 2017, 46, 3185.

[95] J. Liu, C. Wöll, Chem. Soc. Rev. 2017, 46, 5730.

[96] Y.-Y. Wang, S.-M. Chen, R. Haldar, C. Wöll, Z.-G. Gu, J. Zhang, Adv. Mater. Interfaces 2018, 5, 1800985.

[97] D. Sheberla, L. Sun, M. A. Blood-Forsythe, S. Er, C. R. Wade, C. K. Brozek, A. Aspuru-Guzik, M. Dincă, J. Am. Chem. Soc. 2014, 136, 8859.

[98] A. J. Clough, J. M. Skelton, C. A. Downes, A. A. de la Rosa, J. W. Yoo, A. Walsh, B. C. Melot, S. C. Marinescu, J. Am. Chem. Soc. 2017, 139, 10863.

[99] M. Hmadeh, Z. Lu, Z. Liu, F. Gándara, H. Furukawa, S. Wan, V. Augustyn, R. Chang, L. Liao, F. Zhou, E. Perre, V. Ozolins, K. Suenaga, X. Duan, B. Dunn, Y. Yamamto, O. Terasaki, O. M. Yaghi, Chem. Mater. 2012, 24, 3511.

[100] M. E. Foster, K. Sohlberg, M. D. Allendorf, A. A. Talin, J. Phys. Chem. Lett. 2018, 9, 481.

[101] S. Chen, J. Dai, X. C. Zeng, Phys. Chem. Chem. Phys. 2015, 17, 5954.
[102] Y. Cui, J. Yan, Z. Chen, J. Zhang, Y. Zou, Y. Sun, W. Xu, D. Zhu, Adv. Sci. 2019, 6, 1802235.

[103] R. Gutzler, D. F. Perepichka, J. Am. Chem. Soc. 2013, 135, 16585.

[104] T. Ishiwata, Y. Furukawa, K. Sugikawa, K. Kokado, K. Sada, J. Am. Chem. Soc. 2013, 135, 5427.

[105] M. Tsotsalas, J. Liu, B. Tettmann, S. Grosjean, A. Shahnas, Z. Wang, C. Azucena, M. Addicoat, T. Heine, J. Lahann, J. Overhage, S. Bräse, H. Gliemann, C. Wöll, J. Am. Chem. Soc. 2014, 136, 8 .

[106] Z. Wang, A. Błaszczyk, O. Fuhr, S. Heissler, C. Wöll, M. Mayor, Nat. Commun. 2017, 8, 14442 .

[107] O. Shekhah, H. Wang, S. Kowarik, F. Schreiber, M. Paulus, M. Tolan, C. Sternemann, F. Evers, D. Zacher, R. A. Fischer, C. Wöll, J. Am. Chem. Soc. 2007, 129, 15118.

[108] D. Zacher, O. Shekhah, C. Wöll, R. A. Fischer, Chem. Soc. Rev. 2009, 38, 1418

[109] R. Makiura, S. Motoyama, Y. Umemura, H. Yamanaka, O. Sakata, H. Kitagawa, Nat. Mater. 2010, 9, 565.

[110] H. Li, M. Eddaoudi, T. L. Groy, O. M. Yaghi, J. Am. Chem. Soc. 1998, 120, 8571.

[111] U. Mueller, M. Schubert, F. Teich, H. Puetter, K. Schierle-Arndt, J. Pastré, J. Mater. Chem. 2006, 16, 626.

[112] C. G. Carson, K. Hardcastle, J. Schwartz, X. Liu, C. Hoffmann, R. A. Gerhardt, R. Tannenbaum, Eur. J. Inorg. Chem. 2009, 2009, 2338.

[113] H. F. Clausen, R. D. Poulsen, A. D. Bond, M.-A. S. Chevallier, B. B. Iversen, J. Solid State Chem. 2005, 178, 3342.

[114] R. Dovesi, V. R. Saunders, C. Roetti, R. Orlando, C. M. Zicovich-Wilson, F. Pascale, B. Civalleri, K. Doll, N. M. Harrison, I. J. Bush, P. D'Arco, M. Llunell, M. Causà, Y. Noël, L. Maschio, A. Erba, M. Rerat, S. Casassa, CRYSTAL17 User's Manual, University of Turin, Turin, Italy 2017.

[115] R. Haldar, A. Mazel, M. Krstić, Q. Zhang, M. Jakoby, I. A. Howard, B. S. Richards, N. Jung, D. Jacquemin, S. Diring, W. Wenzel, F. Odobel, C. Wöll, Nat. Commun. 2019, 10, 2048.

[116] M. A. Addicoat, D. E. Coupry, T. Heine, J. Phys. Chem. A 2014, 118, 9607.

[117] R. Haldar, M. Jakoby, A. Mazel, Q. Zhang, A. Welle, T. Mohamed, P. Krolla, W. Wenzel, S. Diring, F. Odobel, B. S. Richards, I. A. Howard, C. Wöll, Nat. Commun. 2018, 9, 4332.

[118] M. Adams, M. Kozlowska, N. Baroni, M. Oldenburg, R. Ma, D. Busko, A. Turshatov, G. Emandi, M. O. Senge, R. Haldar, C. Wöll, G. U. Nienhaus, B. S. Richards, I. A. Howard, ACS Appl. Mater. Interfaces 2019, 11, 15688.

[119] R. L. Martin, M. Haranczyk, Cryst. Growth Des. 2014, 14, 2431.

[120] MOFplus, https://www.mofplus.org (accessed: October 2019).

[121] A. K. Rappe, C. J. Casewit, K. S. Colwell, W. A. Goddard, W. M. Skiff, J. Am. Chem. Soc. 1992, 114, 10024.

[122] M. A. Addicoat, N. Vankova, I. F. Akter, T. Heine, J. Chem. Theory Comput. 2014, 10, 880.

[123] D. E. Coupry, M. A. Addicoat, T. Heine, J. Chem. Theory Comput. 2016, 12, 5215

[124] D. E. Coupry, M. A. Addicoat, T. Heine, J. Chem. Phys. 2017, 147, 161705.

[125] S. Bureekaew, S. Amirjalayer, M. Tafipolsky, C. Spickermann, T. K. Roy, R. Schmid, Phys. Status Solidi B 2013, 250, 1128.

[126] P. G. Boyd, S. M. Moosavi, M. Witman, B. Smit, J. Phys. Chem. Lett. 2017, 8, 357.

[127] D. Porezag, T. Frauenheim, T. Köhler, G. Seifert, R. Kaschner, Phys. Rev. B 1995, 51, 12947.

[128] G. Seifert, D. Porezag, T. Frauenheim, Int. J. Quantum Chem. 1996, 58, 185.

[129] M. Elstner, D. Porezag, G. Jungnickel, J. Elsner, M. Haugk, T. Frauenheim, S. Suhai, G. Seifert, Phys. Rev. B 1998, 58, 7260.

[130] M. Gaus, Q. Cui, M. Elstner, J. Chem. Theory Comput. 2011, 7, 931. 
[131] B. Lukose, B. Supronowicz, P. St Petkov, J. Frenzel, A. B. Kuc, G. Seifert, G. N. Vayssilov, T. Heine, Phys. Status Solidi B 2012, 249, 335.

[132] M. Gaus, A. Goez, M. Elstner, J. Chem. Theory Comput. 2013, 9, 338.

[133] C. Köhler, G. Seifert, T. Frauenheim, Chem. Phys. 2005, 309, 23.

[134] P. Melix, A. F. Oliveira, R. Rüger, T. Heine, Theor. Chem. Acc. 2016, 135, 232.

[135] C. Köhler, T. Frauenheim, B. Hourahine, G. Seifert, M. Sternberg, J. Phys. Chem. A 2007, 111, 5622.

[136] L. Zhechkov, T. Heine, S. Patchkovskii, G. Seifert, H. A. Duarte, J. Chem. Theory Comput. 2005, 1, 841

[137] J. G. Brandenburg, S. Grimme, J. Phys. Chem. Lett. 2014, 5, 1785.

[138] M. Mortazavi, J. G. Brandenburg, R. J. Maurer, A. Tkatchenko, J. Phys. Chem. Lett. 2018, 9, 399.

[139] X. Chen, M. Addicoat, S. Irle, A. Nagai, D. Jiang, J. Am. Chem. Soc. 2013, 135, 546.

[140] S. Dalapati, M. Addicoat, S. Jin, T. Sakurai, J. Gao, H. Xu, S. Irle, S. Seki, D. Jiang, Nat. Commun. 2015, 6, 7786.

[141] H. Xu, X. Chen, J. Gao, J. Lin, M. Addicoat, S. Irle, D. Jiang, Chem. Commun. 2014, 50, 1292.

[142] B. P. Biswal, S. Chandra, S. Kandambeth, B. Lukose, T. Heine, R. Banerjee, J. Am. Chem. Soc. 2013, 135, 5328.

[143] S. Kandambeth, A. Mallick, B. Lukose, M. V. Mane, T. Heine, R. Banerjee, J. Am. Chem. Soc. 2012, 134, 19524.

[144] S. Chandra, S. Kandambeth, B. P. Biswal, B. Lukose, S. M. Kunjir, M. Chaudhary, R. Babarao, T. Heine, R. Banerjee, J. Am. Chem. Soc. 2013, 135, 17853.

[145] S. Chandra, T. Kundu, K. Dey, M. Addicoat, T. Heine, R. Banerjee, Chem. Mater. 2016, 28, 1489.

[146] S. Chandra, D. R. Chowdhury, M. Addicoat, T. Heine, A. Paul, R. Banerjee, Chem. Mater. 2017, 29, 2074.

[147] G. Das, B. P. Biswal, S. Kandambeth, V. Venkatesh, G. Kaur, M. Addicoat, T. Heine, S. Verma, R. Banerjee, Chem. Sci. 2015, 6, 3931.

[148] A. Halder, S. Karak, M. Addicoat, S. Bera, A. Chakraborty, S. H. Kunjattu, P. Pachfule, T. Heine, R. Banerjee, Angew. Chem., Int. Ed. 2018, 57, 5797.

[149] A. Halder, S. Kandambeth, B. P. Biswal, G. Kaur, N. C. Roy, M. Addicoat, J. K. Salunke, S. Banerjee, K. Vanka, T. Heine, S. Verma, R. Banerjee, Angew. Chem., Int. Ed. 2016, 55, 7806.

[150] S. Xu, G. Wang, B. P. Biswal, M. Addicoat, S. Paasch, W. Sheng, X. Zhuang, E. Brunner, T. Heine, R. Berger, X. Feng, Angew. Chem., Int. Ed. 2019, 58, 849.

[151] M. Servalli, K. Celebi, P. Payamyar, L. Zheng, M. Položij, B. Lowe, A. Kuc, T. Schwarz, K. Thorwarth, A. Borgschulte, T. Heine, R. Zenobi, A. D. Schlüter, ACS Nano 2018, 12, 11294.

[152] Z. Kang, Y. Peng, Y. Qian, D. Yuan, M. A. Addicoat, T. Heine, Z. Hu, L. Tee, Z. Guo, D. Zhao, Chem. Mater. 2016, 28, 1277.

[153] B. Aradi, B. Hourahine, T. Frauenheim, J. Phys. Chem. A 2007, 111, 5678.

[154] R. Rüger, A. Yakovlev, P. Philipsen, S. Borini, P. Melix, A. F. Oliveira, M. Franchini, T. Soini, M. de Reus, M. G. Asl,
T. Q. Teodoro, D. McCormack, S. Patchkovskii, T. Heine, AMS DFTB 2019: SCM, Theoretical Chemistry, Vrije Universiteit, Amsterdam, The Netherlands.

[155] Y. Nishimoto, D. G. Fedorov, S. Irle, J. Chem. Theory Comput. 2014, 10, 4801.

[156] P. E. Blöchl, Phys. Rev. B 1994, 50, 17953.

[157] The Vienna Ab initio Simulation Package: Atomic scale materials modelling from first principles, https://www.vasp.at (accessed: October 2019).

[158] Castep homepage, http://www.castep.org (accessed: October 2019).

[159] P. Giannozzi, O. Andreussi, T. Brumme, O. Bunau, M. Buongiorno Nardelli, M. Calandra, R. Car, C. Cavazzoni, D. Ceresoli, M. Cococcioni, N. Colonna, I. Carnimeo, A. Dal Corso, S. de Gironcoli, P. Delugas, R. A. DiStasio, A. Ferretti, A. Floris, G. Fratesi, G. Fugallo, R. Gebauer, U. Gerstmann, F. Giustino, T. Gorni, J. Jia, M. Kawamura, H.-Y. Ko, A. Kokalj, E. Küçükbenli, M. Lazzeri, M. Marsili, N. Marzari, F. Mauri, N. L. Nguyen, H.-V. Nguyen, A. Otero-de-la-Roza, L. Paulatto, S. Poncé, D. Rocca, R. Sabatini, B. Santra, M. Schlipf, A. P. Seitsonen, A. Smogunov, I. Timrov, T. Thonhauser, P. Umari, N. Vast, X. Wu, S. Baroni, J. Phys.: Condens. Matter 2017, 29, 465901.

[160] About CP2K, https://www.cp2k.org (accessed: October 2019).

[161] FHI aims, https://aimsclub.fhi-berlin.mpg.de (accessed: October 2019).

[162] C. Adamo, V. Barone, J. Chem. Phys. 1999, 110, 6158.

[163] J. Heyd, J. E. Peralta, G. E. Scuseria, R. L. Martin, J. Chem. Phys. 2005, 123, 174101.

[164] S. Grimme, J. Antony, S. Ehrlich, H. Krieg, J. Chem. Phys. 2010, $132,154104$.

[165] A. Tkatchenko, R. A. DiStasio, R. Car, M. Scheffler, Phys. Rev. Lett. 2012, 108, 236402.

[166] J. P. Perdew, K. Burke, M. Ernzerhof, Phys. Rev. Lett. 1996, 77, 3865.

[167] E. van Lenthe, J. G. Snijders, E. J. Baerends, J. Chem. Phys. 1996, 105,6505 .

[168] M. Kuisma, J. Ojanen, J. Enkovaara, T. T. Rantala, Phys. Rev. B 2010, 82, 115106.

[169] O. Gritsenko, R. van Leeuwen, E. van Lenthe, E. J. Baerends, Phys. Rev. A 1995, 51, 1944.

[170] M. L. Cohen, S. G. S. Louie, Fundamentals of Condensed Matter Physics, Cambridge University Press, Cambridge, UK 2016.

[171] J.-L. Zhuang, A. Terfort, C. Wöll, Coord. Chem. Rev. 2016, 307, 391.

[172] H. K. Arslan, O. Shekhah, J. Wohlgemuth, M. Franzreb, R. A. Fischer, C. Wöll, Adv. Funct. Mater. 2011, 21, 4228.

[173] Z. Wang, J. Liu, B. Lukose, Z. Gu, P. G. Weidler, H. Gliemann, T. Heine, C. Wöll, Nano Lett. 2014, 14, 1526.

[174] O. Shekhah, K. Hirai, H. Wang, H. Uehara, M. Kondo, S. Diring, D. Zacher, R. A. Fischer, O. Sakata, S. Kitagawa, S. Furukawa, C. Wöll, Dalton Trans. 2011, 40, 4954.

[175] D. Chen, R. Haldar, B. L. Neumeier, Z. Fu, C. Feldmann, C. Wöll, E. Redel, Adv. Funct. Mater. 2019, 29, 1903086. 